\title{
Optics of Inhomogeneous Thin Films with Defects: Application to Optical Characterization
}

\author{
Ivan Ohlídal *, Jiří Vohánka (D) and Martin Čermák
}

Department of Physical Electronics, Faculty of Science, Masaryk University, Kotlářská 2, 61137 Brno, Czech Republic; vohanka@physics.muni.cz (J.V.); 63855@seznam.cz (M.Č.)

* Correspondence: ohlidal@physics.muni.cz

check for updates

Citation: Ohlídal, I.; Vohánka, J.; Čermák, M. Optics of Inhomogeneous Thin Films with Defects: Application to Optical Characterization. Coatings 2021, 11, 22. https://doi.org/10.3390/ coatings11010022

Received: 30 November 2020 Accepted: 21 December 2020 Published: 27 December 2020

Publisher's Note: MDPI stays neutral with regard to jurisdictional claims in published maps and institutional affiliations.

Copyright: (c) 2020 by the authors. Licensee MDPI, Basel, Switzerland. This article is an open access article distributed under the terms and conditions of the Creative Commons Attribution (CC BY) license (https://creativecommons.org/ licenses/by/4.0/).

\begin{abstract}
This review paper is devoted to optics of inhomogeneous thin films exhibiting defects consisting in transition layers, overlayers, thickness nonuniformity, boundary roughness and uniaxial anisotropy. The theoretical approaches enabling the inclusion of these defects into formulae expressing the optical quantities of these inhomogeneous thin films are summarized. These approaches are based on the recursive and matrix formalisms for the transition layers and overlayers, averaging of the elements of the Mueller matrix using local thickness distribution or polynomial formulation for the thickness nonuniformity, scalar diffraction theory and Rayleigh-Rice theory or their combination for boundary roughness and Yeh matrix formalism for uniaxial anisotropy. The theoretical results are illustrated using selected examples of the optical characterization of the inhomogeneous polymerlike thin films exhibiting the combination of the transition layers and thickness nonuniformity and inhomogeneous thin films of nonstoichiometric silicon nitride with the combination of boundary roughness and uniaxial anisotropy. This characterization is realized by variable angle spectroscopic ellipsometry and spectroscopic reflectometry. It is shown that using these optical techniques, the complete optical characterization of the mentioned thin films can be performed. Thus, it is presented that the values of all the parameters characterizing these films can be determined.
\end{abstract}

Keywords: optical characterization; ellipsometry; reflectometry inhomogeneous films

\section{Introduction}

Optics of thin films represents an important field in fundamental and applied studies. Homogeneous and inhomogeneous thin films occur in both fields. Homogeneous thin films exhibit an identical complex refractive index within the whole volumes of these films. A complex refractive index of inhomogeneous thin films changes inside these films. Most inhomogeneous thin films exhibit profiles of the complex refractive index along the axis perpendicular to their parallel boundaries. These profiles are mostly represented by continuous complex functions. Many studies of the optical properties of homogeneous and inhomogeneous thin films have been performed so far. These studies are realized using the optical characterization of the films mentioned. An enormous attention has been devoted to developing methods of the optical characterization of homogeneous thin films (see e.g., [1-19]). Less attention has been devoted to the optical characterization of inhomogeneous thin films [20-25]. However, in the three last decades researchers dealt with the optical characterization of inhomogeneous thin films more intensively. This is caused by developments in microelectronics, semiconductor industry, solar energetics, etc. Thin films with complex structures, which are frequently inhomogeneous from the optical point of view, often occur in these branches. An example of such inhomogeneous thin films consisting of complex materials are inhomogeneous thin films of nonstoichiometric silicon nitride $\left(\mathrm{SiN}_{x}\right)$ that are rich in silicon (see e.g., [26]). Moreover, in the optics industry layered systems consisting of thin films with different refractive indices are sometimes replaced by suitable inhomogeneous thin films. This is caused by better optical properties 
of these inhomogeneous thin films in comparison with the corresponding layered systems. For example, the inhomogeneous thin films with continuous refractive index profiles exhibit substantially lower light scattering caused by boundary roughness than the layered systems. The example of such an inhomogeneous thin film replacing the layered systems are rugate filters (see e.g., $[27,28])$. Therefore, new efficient methods for the optical characterization of the inhomogeneous thin films had to be developed. These methods are presented, for example, in papers [29-34].

Homogeneous and inhomogeneous thin films often exhibit various defects. These defects must be taken into account at their optical characterization. A considerable effort has been devoted to the optical characterization of homogeneous thin films exhibiting defects. Methods of the optical characterization of the homogeneous thin films having random roughness of their boundaries were presented in papers [35-48]. Thickness nonuniformity is also a defect often exhibited by the homogeneous thin films. Several spectroellipsometric and spectrophotometric methods enabling the complete optical characterization of the homogeneous thin films with this defect were presented in the literature [49-57]. Thickness nonuniformity can also be measured by means of imagining spectroscopic reflectometry (ISR). Using this technique the maps of spectral dependencies of local reflectance at normal incidence are measured along surfaces of samples (CCD cameras are detectors of imaging reflectometers). These local reflectance maps allow for the determination of maps of local thicknesses of the nonuniform homogeneous thin films. This technique is especially efficient if the optical constants of the films characterized are known or determined independently. The methods of ISR usable for the optical characterization of nonuniform homogeneous thin films are presented in papers [58-61]. Transition layers at the boundaries of thin films also represent one of the defects. These layers are usually very thin and, sometimes, they are also inhomogeneous. Therefore, it can be difficult to include them in the optical characterization of thin films. Methods enabling the optical characterization of some homogeneous thin films containing transition layers are presented, for example, in [62-64]. It should be noted that the successful optical characterization can be carried out for the homogeneous thin films exhibiting a combination of defects. For example, the complete optical characterization of epitaxial ZnSe thin films exhibiting a combination of a thickness nonuniformity and random roughness of their upper boundaries was performed in paper [65].

Sufficient attention has not been devoted to the optical characterization of inhomogeneous thin films exhibiting defects so far. This is caused by difficult theoretical approaches needed for realizing the optical characterization of these films. The formulae for optical quantities of the inhomogeneous thin films with defects are more complicated than those corresponding to homogeneous thin films exhibiting the same defects. This implies that a numerical processing of experimental data is also more complicated for these inhomogeneous thin films because it is necessary to search a larger number of parameters describing them.

To our knowledge only three papers have been devoted to the complete optical characterization of inhomogeneous thin films exhibiting defects so far. In paper [66] the optical characterization of inhomogeneous thin films of $\mathrm{SiN}_{x}$ and $\mathrm{SiO}_{x} \mathrm{C}_{y} \mathrm{H}_{z}$ containing transition layers at their lower boundaries is performed. In paper [67] inhomogeneous thin films of $\mathrm{SiN}_{x}$ with random roughness of the upper boundaries and unwanted uniaxial anisotropy are characterized. In paper [68] the complete optical characterization of $\mathrm{SiO}_{x} \mathrm{C}_{y} \mathrm{H}_{z}$ thin films exhibiting transition layers at the lower boundaries and wedge-shaped thickness nonuniformity is carried out.

In this paper a review of new theoretical and experimental results concerning optics and the optical characterization of inhomogeneous thin films exhibiting defects consisting in thickness nonuniformity, boundary roughness, transition layers, overlayers and unwanted artificial uniaxial anisotropy achieved during the several last years will be presented. This means that the formulae for optical quantities of inhomogeneous thin films with the mentioned defects describing their optical properties will be presented. Two 
examples of the optical characterization of selected inhomogeneous thin films with defects will be introduced as well. Our attention will be devoted to light reflected from these films.

The organization of our paper is as follows: The paper is divided into four basic sections, i.e., Introduction, Theory, Examples of optical characterization and Conclusion.

The theory section is divided into two parts. The first part contains the description of approximate theoretical approaches usable for the derivation of formulae for the optical quantities of inhomogeneous thin films without defects. The second part of this section describes the theoretical approaches by means of which the formulae for the optical quantities of the inhomogeneous thin films exhibiting defects are calculated. The following defects are taken into account: transition layers, overlayers, thickness nonuniformity, random roughness of boundaries and artificial uniaxial anisotropy.

Section Examples of optical characterization comprises the results obtained within the optical characterization of the inhomogeneous polymer-like thin film exhibiting the combination of two defects, i.e., transition layer at the lower boundary of this film and complicated thickness nonuniformity. Moreover, this section contains the results of the optical characterization of three inhomogeneous thin films of nonstoichiometric silicon nitride with the combination of random roughness of the upper boundaries of these films and artificial uniaxial anisotropy.

In the conclusion, the theoretical and experimental results presented in this review paper are summarized and discussed.

\section{Theory}

\subsection{Inhomogeneous thin Films without Defects}

Exact solutions of an interaction of light with ideal homogeneous thin films, i.e., films without defects, are known only for several types of refractive index profiles. These profiles are presented in $[69,70]$. Concrete solutions corresponding to them, i.e., formulae for the optical quantities, are published in [69,71]. It should be noted that the use of these exact solutions is rather limited in practice since most real inhomogeneous thin films exhibit the profiles of the refractive index differing from those with the exact solutions. Therefore, approximate methods are mostly utilized for calculating the optical quantities of these inhomogeneous thin films. These approximate methods will be briefly described in this section.

\subsubsection{Wentzel-Kramers-Brillouin-Jeffreys (WKBJ) Method}

This approximation can be used for the optical characterization of the inhomogeneous thin films whose gradients of the refractive index profiles are very small. In this case, the reflections occurring inside the inhomogeneous thin films can be neglected and the optical quantities of these films depend only on the refractive indices at the upper and lower boundaries and the optical path lengths. The reflection coefficients $r_{0}$ are expressed as follows $[69,70]$ :

$$
r_{0}=\frac{r_{\mathrm{U}}+r_{\mathrm{L}} \mathrm{e}^{\mathrm{i} x_{d}}}{1+r_{\mathrm{U}} r_{\mathrm{L}} \mathrm{e}^{\mathrm{i} x_{d}}},
$$

where

$$
r_{\mathrm{U}}=\frac{Y_{\mathrm{A}}-Y_{\mathrm{U}}}{Y_{\mathrm{A}}+Y_{\mathrm{U}}}, \quad r_{\mathrm{L}}=\frac{Y_{\mathrm{L}}-Y_{\mathrm{S}}}{Y_{\mathrm{L}}+Y_{\mathrm{S}}}, \quad x_{d}=\frac{4 \pi}{\lambda} \int_{0}^{d} \sqrt{n^{2}(z)-n_{\mathrm{A}}^{2} \sin ^{2} \varphi_{\mathrm{A}}} \mathrm{d} z .
$$

Symbols $d, n_{\mathrm{A}}, \varphi_{\mathrm{A}}$ and $\lambda$ represent the thickness, refractive index of the ambient, incidence angle of light on the upper boundary and wavelength of incident light, respectively. These quantities are always real. The other quantities are complex in general. Symbols $r_{0}$, $r_{\mathrm{U}}$ and $r_{\mathrm{L}}$ are the reflection coefficients of the film, upper boundary and lower boundary for both the polarization $q=\mathrm{p}$, s, where $\mathrm{p}$ and $\mathrm{s}$ denote the p-polarization and s-polarization, respectively. In order to make the formulae shorter, the indices $q$, $\mathrm{p}$ and $\mathrm{s}$ will be omitted wherever possible. They will be used only in formulae where their omission is not possible, 
or if their omission could cause confusion. Symbol $n(z)$ denotes a function describing refractive index profile of the inhomogeneous thin film (the coordinate $z$ corresponds to the axis perpendicular to the parallel boundaries or the parallel mean planes of these boundaries). Symbols $Y_{\mathrm{A}}, Y_{\mathrm{U}}, Y_{\mathrm{L}}$ and $Y_{\mathrm{S}}$ represent the optical admittances of the ambient, at the upper boundary, at the lower boundary and of the substrate for both the polarizations, respectively. The following equations are valid for the admittances corresponding to the p-polarization and s-polarization:

$$
Y_{i, \mathrm{p}}=\frac{n_{i}}{\cos \varphi_{i}}, \quad Y_{i, \mathrm{~s}}=n_{i} \cos \varphi_{i}
$$

where index $i=A, \mathrm{U}, \mathrm{L}, \mathrm{S}$. Symbols $\varphi_{\mathrm{U}}, \varphi_{\mathrm{L}}$ and $\varphi_{\mathrm{S}}$ denote the refraction angles at the upper boundary, lower boundary and substrate, respectively. These angles together with the angle of incidence fulfill the Snell's law. The reflection coefficients $r_{0}$ enable us to calculate the reflectance and ellipsometric quantities of the films.

\subsubsection{Approximate Method Based on Using Multilayer Systems}

This approximation can be used to calculate the optical quantities of the inhomogeneous thin films having larger gradients of the refractive index profiles, i.e., when the WKBJ approximation is not usable. This method is based on replacing the inhomogeneous thin films by multilayer systems consisting of a large number of homogeneous layers exhibiting very small thicknesses and very small differences in refractive indices of the adjacent layers. The reflection coefficients $r$ of such the inhomogeneous thin films are given by the following recursive formulae (see e.g., [72,73]):

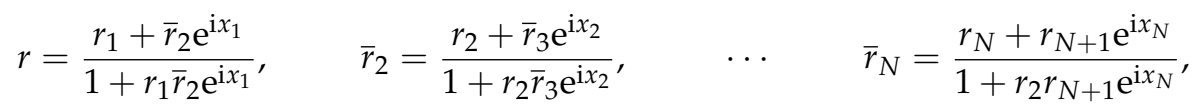

where

$$
\begin{aligned}
& r_{1}=\frac{Y_{\mathrm{A}}-Y_{1}}{Y_{\mathrm{A}}+Y_{1}}, \quad r_{2}=\frac{Y_{1}-Y_{2}}{Y_{1}+Y_{2}}, \quad \ldots \quad r_{N}=\frac{Y_{N-1}-Y_{N}}{Y_{N-1}+Y_{N}}, \quad r_{N+1}=\frac{Y_{N}-Y_{\mathrm{S}}}{Y_{N}+Y_{\mathrm{S}}}, \\
& x_{j}=\frac{4 \pi d_{j}}{\lambda} \sqrt{n_{j}^{2}-n_{\mathrm{A}}^{2} \sin ^{2} \varphi_{\mathrm{A}}},
\end{aligned}
$$

where symbols $d_{j}, n_{j}$ and $Y_{j}$ represent thickness, refractive index and admittances of the individual layers, respectively $(j=1,2, \ldots, N)$. Symbol $\bar{r}$ denotes the effective reflection coefficients corresponding to the system consisting of $(N-j)$ layers on top of the substrate [72-74]. It should be noted that the reflection coefficients of this multilayer system can also be efficiently calculated using the matrix formalism (see e.g., [73]). Note that in paper [33] an improvement of the presented method is carried out. The exact results for the inhomogeneous thin film correspond to the limit in which the number $N$ of approximating layers goes to infinity, i.e., thicknesses of the individual layers go to zero. The improvement presented in [33] consists in using the Richardson extrapolation to increase the convergence to the exact result. If the Richardson extrapolation is utilized the error falls approximately as $N^{-\log _{2} N}$. However, it is necessary to point out that the faster convergence is achieved only if the number of the approximating layers is very large (for details see [33]).

2.1.3. Approximate Method Based on Modification of Recursive Formulae of Multilayer Systems

In paper [75], the efficient approximate method for expressing the formulae describing the optical quantities of the inhomogeneous thin films is presented. This method is based on the modification of the recursive formalism for the reflection coefficients of multilayer systems. In the derivation of the formulae for the reflection coefficients, the four-layer system is utilized. This system is subdivided in more and more sublayers. Then the limit for infinite number of the sublayers enables one to write the formulae for the reflection 
coefficients of the inhomogeneous thin films with any profiles of the refractive indices. In the derived formulae, the multiple integrals occur. The multiplicity of the integrals correspond to numbers of internal reflections inside the inhomogeneous thin film. If the single reflections inside the film are sufficient then the single integrals are taken into account. In this case the following formulae for the reflection coefficients of the inhomogeneous thin films is valid:

$$
r=\frac{r_{\mathrm{U}}+B_{1}+r_{\mathrm{U}} r_{L} B_{2} \mathrm{e}^{\mathrm{i} x_{d}}+r_{\mathrm{L}} \mathrm{e}^{\mathrm{i} x_{d}}}{1+r_{\mathrm{U}} B_{1}+r_{\mathrm{L}} B_{2} \mathrm{e}^{\mathrm{i} x_{d}}+r_{\mathrm{U}} r_{\mathrm{L}} \mathrm{e}^{\mathrm{i} x_{d}}}
$$

where

$$
\begin{aligned}
B_{1} & =\int_{0}^{d} f\left(z_{1}\right) \mathrm{e}^{\mathrm{i} x\left(z_{1}\right)} \mathrm{d} z_{1}, & B_{2} & =\int_{0}^{d} f\left(z_{1}\right) \mathrm{e}^{-\mathrm{i} x\left(z_{1}\right)} \mathrm{d} z_{1}, \\
x\left(z_{1}\right) & =\frac{4 \pi}{\lambda} \int_{0}^{z_{1}} \sqrt{n^{2}(z)-n_{\mathrm{A}}^{2} \sin ^{2} \varphi_{\mathrm{A}}} \mathrm{d} z, & f\left(z_{1}\right) & =\frac{1}{2 Y\left(z_{1}\right)} \frac{\mathrm{d} Y\left(z_{1}\right)}{\mathrm{d} z_{1}} .
\end{aligned}
$$

In paper [75], the formulae for the reflection coefficients of the film corresponding to double and triple integrals are introduced. The formulae presented in [75] can be used to calculate the reflectance and ellipsometric parameters of the inhomogeneous thin films with larger gradients of refractive indices in a reliable way. Moreover, the matrix formalism for expressing the reflection coefficients of the considered films are also presented in this paper.

\subsubsection{Approximate Method Based on Multiple-Beam Interference Model}

Within this method the derivation of the formula for the reflection coefficients of the inhomogeneous thin films is based on calculations of corrections to the WKBJ formula corresponding to multiple reflections inside these films. The corrections contain multiple integrals similar to those presented in [75]. A multiplicity of the integrals again corresponds to the number of the internal reflection inside the films. After employing the mathematical approach presented in paper [76], one obtains the following formula for the reflection coefficients of the films considered:

$$
r=r_{0}+\sum_{n=1}^{M} \Delta r_{n}
$$

where

$$
\Delta r_{n}=\sum_{l=1}^{3^{n-1}}\left(I_{n}^{(l)}+\bar{I}_{n}^{(l)}\right) .
$$

Symbols $\Delta r_{n}, I_{n}^{(l)}, \bar{I}_{n}^{(l)}$ and $M$ denote the total correction of the $n$-th order, partial corrections of the $n$-th order and maximum order, respectively. The partial corrections of the first order are expressed as follows:

$$
I_{1}^{(1)}=C_{1}^{(1)} j_{1}^{(1)}, \quad \bar{I}_{1}^{(1)}=\bar{C}_{1}^{(1)} \bar{j}_{1}^{(1)},
$$

where

$$
\begin{aligned}
& C_{1}^{(1)}=\frac{\tau \mathrm{e}^{\mathrm{i} x_{d}}}{(1-\varrho)^{2}}, \quad j_{1}^{(1)}=\int_{0}^{d} f\left(z_{1}\right) \mathrm{e}^{-\mathrm{i} x\left(z_{1}\right)} \mathrm{d} z_{1}, \\
& \bar{C}_{1}^{(1)}=-\frac{\tau r_{\mathrm{L}} Z}{(1-\varrho)^{2}}, \quad \bar{j}_{1}^{(1)}=\int_{0}^{d} f\left(z_{1}\right) \mathrm{e}^{\mathrm{i} x\left(z_{1}\right)} \mathrm{d} z_{1}, \\
& \mathrm{Z}=r_{\mathrm{L}} \mathrm{e}^{\mathrm{i} x_{d}}, \quad \varrho=-r_{\mathrm{U}} r_{\mathrm{L}} \mathrm{e}^{\mathrm{i} x_{d}}, \quad \tau=t_{\mathrm{U}} t_{\mathrm{U}}^{\prime} .
\end{aligned}
$$


The transmission coefficients $t_{\mathrm{U}}$ and $t_{\mathrm{U}}^{\prime}$ are given as

$$
t_{\mathrm{U}}=\frac{2 Y_{\mathrm{A}}}{Y_{\mathrm{A}}+Y_{\mathrm{U}}}, \quad t_{\mathrm{U}}^{\prime}=\frac{2 Y_{\mathrm{U}}}{Y_{\mathrm{A}}+Y_{\mathrm{U}}},
$$

for the s polarization and

$$
t_{\mathrm{U}}=\frac{\cos \varphi_{\mathrm{A}}}{\cos \varphi_{\mathrm{U}}} \frac{2 Y_{\mathrm{A}}}{Y_{\mathrm{A}}+Y_{\mathrm{U}}}, \quad t_{\mathrm{U}}^{\prime}=\frac{\cos \varphi_{\mathrm{U}}}{\cos \varphi_{\mathrm{A}}} \frac{2 Y_{\mathrm{U}}}{Y_{\mathrm{A}}+Y_{\mathrm{U}}},
$$

for the p polarization.

The partial corrections of the $n$-th order are given as

$$
I_{n}^{(l)}=C_{n}^{(l)} j_{n}^{(l)}, \quad \bar{I}_{n}^{(l)}=\bar{C}_{n}^{(l)} \bar{j}_{n}^{(l)},
$$

where the quantities $j_{n}^{(l)}$ and $\bar{j}_{n}^{(l)}$ are given by $n$-dimensional integrals and the quantities $C_{n}^{(l)}$ and $\bar{C}_{n}^{(l)}$ represent factors given by some commbination of the Fresnel coefficients at the boundaries and the term $\mathrm{e}^{\mathrm{i} x_{d}}$. The concrete form of the partial corrections can be determined using the recursive procedure derived in [76]. Within this procedure, each partial correction $I_{n}^{(l)}$ of $n$-th order gives three partial corrections $I_{n+1}^{(3 l-2)}, I_{n+1}^{(3 l-1)}, I_{n+1}^{(3 l)}$ of $(n+1)$-th order. It is necessary to distinguish two cases. The first case corresponds to the partial correction of the $n$-th order given by the integral $j_{n}^{(l)}$ in the following form

$$
j_{n}^{(l)}=\int_{0}^{d} \cdots \int_{\alpha}^{\beta}\left[\prod_{v=1}^{n} f\left(z_{v}\right)\right] \mathrm{e}^{-\mathrm{i} x\left(z_{1}\right)} \cdots \mathrm{e}^{-\mathrm{i} x\left(z_{n}\right)} \mathrm{d} z_{n} \cdots \mathrm{d} z_{1},
$$

where $\alpha=0, \beta=d$ or $\alpha=0, \beta=z_{n-1}$. The important criterion is that the last term in the integral is $\mathrm{e}^{-\mathrm{i} x\left(z_{n}\right)}$, which distinguishes it from the other case with $\mathrm{e}^{\mathrm{i} x\left(z_{n}\right)}$. The partial corrections of the $(n+1)$-th order are then defined by the following formulae

$$
\begin{aligned}
C_{n+1}^{(3 l-2)} & =-\frac{r_{\mathrm{U}} \mathrm{e}^{\mathrm{i} x_{d}}}{1-\varrho} C_{n}^{(l)}, \\
j_{n+1}^{(3 l-2)} & =\int_{0}^{d} \cdots \int_{\alpha}^{\beta} \int_{0}^{d}\left[\prod_{v=1}^{n+1} f\left(z_{v}\right)\right] \mathrm{e}^{-\mathrm{i} x\left(z_{1}\right)} \cdots \mathrm{e}^{-\mathrm{i} x\left(z_{n}\right)} \mathrm{e}^{-\mathrm{i} x\left(z_{n+1}\right)} \mathrm{d} z_{n+1} \cdots \mathrm{d} z_{1}, \\
C_{n+1}^{(3 l-1)} & =-r_{\mathrm{L}} C_{n}^{(l)}, \\
j_{n+1}^{(3 l-1)} & =\int_{0}^{d} \cdots \int_{\alpha}^{\beta} \int_{z_{n}}^{d}\left[\prod_{v=1}^{n+1} f\left(z_{v}\right)\right] \mathrm{e}^{-\mathrm{i} x\left(z_{1}\right)} \cdots \mathrm{e}^{-\mathrm{i} x\left(z_{n}\right)} \mathrm{e}^{\mathrm{i} x\left(z_{n+1}\right)} \mathrm{d} z_{n+1} \cdots \mathrm{d} z_{1}, \\
C_{n+1}^{(3 l)} & =-\frac{r_{\mathrm{L}} \varrho}{1-\varrho} C_{n}^{(l)}, \\
j_{n+1}^{(3 l)} & =\int_{0}^{d} \cdots \int_{\alpha}^{\beta} \int_{0}^{d}\left[\prod_{v=1}^{n+1} f\left(z_{v}\right)\right] \mathrm{e}^{-\mathrm{i} x\left(z_{1}\right)} \cdots \mathrm{e}^{-\mathrm{i} x\left(z_{n}\right)} \mathrm{e}^{\mathrm{i} x\left(z_{n+1}\right)} \mathrm{d} z_{n+1} \cdots \mathrm{d} z_{1},
\end{aligned}
$$

The second case corresponds to the integral $j_{n}^{(l)}$ given in the following form

$$
j_{n}^{(l)}=\int_{0}^{d} \cdots \int_{\alpha}^{\beta}\left[\prod_{v=1}^{n} f\left(z_{v}\right)\right] \mathrm{e}^{-\mathrm{i} x\left(z_{1}\right)} \cdots \mathrm{e}^{\mathrm{i} x\left(z_{n}\right)} \mathrm{d} z_{n} \cdots \mathrm{d} z_{1},
$$


where $\alpha=0, \beta=d$ or $\alpha=z_{n-1}, \beta=d$. The partial corrections of the $(n+1)$-th order are defined by the following formulae

$$
\begin{aligned}
C_{n+1}^{(3 l-2)} & =\frac{1}{r_{\mathrm{L}}} C_{n}^{(l)}, \\
j_{n+1}^{(3 l-2)} & =\int_{0}^{d} \cdots \int_{\alpha}^{\beta} \int_{0}^{z_{n}}\left[\prod_{v=1}^{n+1} f\left(z_{v}\right)\right] \mathrm{e}^{-\mathrm{i} x\left(z_{1}\right)} \cdots \mathrm{e}^{\mathrm{i} x\left(z_{n}\right)} \mathrm{e}^{-\mathrm{i} x\left(z_{n+1}\right)} \mathrm{d} z_{n+1} \cdots \mathrm{d} z_{1}, \\
C_{n+1}^{(3 l-1)} & =-\frac{r_{\mathrm{U}} \mathrm{e}^{\mathrm{i} x_{d}}}{1-\varrho} C_{n}^{(l)}, \\
j_{n+1}^{(3 l-1)} & =\int_{0}^{d} \cdots \int_{\alpha}^{\beta} \int_{0}^{d}\left[\prod_{v=1}^{n+1} f\left(z_{v}\right)\right] \mathrm{e}^{-\mathrm{i} x\left(z_{1}\right)} \cdots \mathrm{e}^{\mathrm{i} x\left(z_{n}\right)} \mathrm{e}^{-\mathrm{i} x\left(z_{n+1}\right)} \mathrm{d} z_{n+1} \cdots \mathrm{d} z_{1}, \\
C_{n+1}^{(3 l)} & =-\frac{r_{\mathrm{L}}}{1-\varrho} C_{n}^{(l)}, \\
j_{n+1}^{(3 l)} & =\int_{0}^{d} \cdots \int_{\alpha}^{\beta} \int_{0}^{d}\left[\prod_{v=1}^{n+1} f\left(z_{v}\right)\right] \mathrm{e}^{-\mathrm{i} x\left(z_{1}\right)} \cdots \mathrm{e}^{\mathrm{i} x\left(z_{n}\right)} \mathrm{e}^{\mathrm{i} x\left(z_{n+1}\right)} \mathrm{d} z_{n+1} \cdots \mathrm{d} z_{1},
\end{aligned}
$$

The foregoing equations represent recursive formulae enabling to derive the formulae for the partial corrections of an arbitrary order. The same recursive formulae are valid for partial corrections $\bar{I}_{n}^{(l)}$. However, in these recursive formulae the exponential factor $\mathrm{e}^{-\mathrm{i} x\left(z_{1}\right)}$ must be systematically replaced by the exponential factor $\mathrm{e}^{\mathrm{i} x\left(z_{1}\right)}$. Moreover, the coefficients $\bar{C}_{n}^{(l)}$ must be generated from the coefficient $\bar{C}_{1}^{(1)}$. The integrals occurring in the foregoing equations can be calculated using the numerical method based on the Chebysev interpolation in an efficient way [76]. In principle this approach can be used to calculate the optical quantities of the inhomogeneous thin films with arbitrary refractive index profiles.

Note that the recursive formulae for the partial corrections written in (15) and (17) are somewhat formally different from those presented in paper [76] because they use the opposite orientation of the axis $z$.

\subsection{Inhomogeneous Thin Films with Defects}

In this section, the methods for including the influence of the individual defects mentioned above on the reflection coefficients of the inhomogeneous thin films are formulated.

\subsubsection{Transition Layers and Overlayers}

Transition layers mostly occur at the lower boundaries of the inhomogeneous thin films, i.e., at boundaries between the substrates and these films. If it is assumed that the transition layers are homogeneous, then the reflection coefficients of the lower boundaries $r_{\mathrm{L}}$ occurring in the formulae presented above (see e.g., Equation (10)) are expressed as follows:

$$
r_{\mathrm{L}}=\frac{r_{\mathrm{L}, \mathrm{T}}+r_{\mathrm{T}, \mathrm{S}} \mathrm{e}^{\mathrm{i} x_{\mathrm{T}}}}{1+r_{\mathrm{L}, \mathrm{T}} r_{\mathrm{T}, \mathrm{S}} \mathrm{e}^{\mathrm{i} x_{\mathrm{T}}}}
$$

where

$$
r_{\mathrm{L}, \mathrm{T}}=\frac{Y_{\mathrm{L}}-Y_{\mathrm{T}}}{Y_{\mathrm{L}}+Y_{\mathrm{T}}}, \quad r_{\mathrm{T}, \mathrm{S}}=\frac{Y_{\mathrm{T}}-Y_{\mathrm{S}}}{Y_{\mathrm{T}}+Y_{\mathrm{S}}}, \quad x_{\mathrm{T}}=\frac{4 \pi}{\lambda} n_{\mathrm{T}} d_{\mathrm{T}} \cos \varphi_{\mathrm{T}} .
$$

Symbols $Y_{\mathrm{T}}, d_{\mathrm{T}}$ and $n_{\mathrm{T}}$ denote the admittance, thickness and refractive index of the transition layer, respectively. 
The reflection coefficients $r$ of the inhomogeneous thin films having the overlayers obey the following equation:

$$
r=\frac{r_{\mathrm{A}, \mathrm{V}}+\bar{r} \mathrm{e}^{\mathrm{i} x_{\mathrm{V}}}}{1+r_{\mathrm{A}, \mathrm{V}} \bar{r} \mathrm{e}^{\mathrm{i} x_{\mathrm{V}}}}
$$

where

$$
r_{\mathrm{A}, \mathrm{V}}=\frac{Y_{\mathrm{A}}-Y_{\mathrm{V}}}{Y_{\mathrm{A}}+Y_{\mathrm{V}}}, \quad x_{\mathrm{V}}=\frac{4 \pi}{\lambda} n_{\mathrm{V}} d_{\mathrm{V}} \cos \varphi_{\mathrm{V}}
$$

Symbols $Y_{\mathrm{V}}, d_{\mathrm{V}}$ and $n_{\mathrm{V}}$ represent the admittance, thickness and refractive index of the overlayer, respectively. Symbol $\bar{r}$ denotes the reflection coefficient of the inhomogeneous thin film calculated by Equation (8) taking into account that $n_{\mathrm{A}}=n_{\mathrm{V}}$.

\subsubsection{Thickness Nonuniformity}

The thickness nonuniformity represents defect frequently occurring in practice. The upper boundaries of thin films exhibiting nonuniformity in thickness are formed by surfaces differing from the planes parallel with the planes of the lower boundaries. Thus, local thicknesses $d(x, y)$ are different along the lower boundary. It is possible to distinguish two basic cases of this nonuniformity in principle. First, the upper boundaries are represented by simple surfaces from the geometrical point of view. A wedge-shaped thickness nonuniformity is the example of such the nonuniformity. Second, the thickness nonuniformity has a complicated form, i.e., the surfaces of the upper boundaries differ from the wedge-shaped nonuniformity or similar simple nonuniformities.

If the wedge-shaped thickness nonuniformity and the circular cross-section of the ellipsometer beam is considered, the following approach based on the local thickness distribution density $\varrho(d)$ can be utilized. Using the reflection coefficients $r$ given by Equation (8) one can calculate the reflectance of the inhomogeneous nonuniform thin films for the p-polarization $R_{\mathrm{p}}$ and s-polarization $R_{\mathrm{s}}$ at an oblique incidence. However, the constant thickness $d$ must be replaced by the function $d(x, y)$ in the upper bounds of the integrals occurring in Section 2.1.4. For example, it is necessary to write

$$
x_{d}=\frac{4 \pi}{\lambda} \int_{0}^{d(x, y)} \sqrt{n^{2}(z)-n_{\mathrm{A}}^{2} \sin ^{2} \varphi_{\mathrm{A}}} \mathrm{d} z, \quad j_{1}^{(1)}=\int_{0}^{d(x, y)} f\left(z_{1}\right) \mathrm{e}^{-\mathrm{i} x\left(z_{1}\right)} \mathrm{d} z_{1},
$$

etc. Function $d(x, y)$ describes the local thicknesses along illuminated spot ( $x$ and $y$ are coordinates in the plane of the lower boundary). Measured reflectances $R_{\mathrm{p}}$ and $R_{\mathrm{s}}$ for the p-polarization and s-polarization, respectively, correspond to the mean values of these quantities, i.e., $\left\langle R_{\mathrm{p}}\right\rangle$ and $\left\langle R_{\mathrm{s}}\right\rangle$. These mean values are given as follows:

$$
\left\langle R_{q}\right\rangle=\int\left|r_{q}(d)\right|^{2} \varrho(d) \mathrm{d} d
$$

where $d \equiv d(x, y)$, symbol $r_{q}(d)$ denotes the reflection coefficients of the inhomogeneous films given by Equation (8) containing local thicknesses $d(x, y)$ in the upper bounds of the integrals and $\varrho(d)$ is the distribution function of the elliptic illuminated spot given in this way (see e.g., [52,70]:

$$
\varrho(d)=\frac{1}{2 \pi \sigma^{2}\left(\varphi_{\mathrm{A}}\right)}\left[4 \sigma^{2}\left(\varphi_{\mathrm{A}}\right)-(d-\bar{d})^{2}\right]^{1 / 2}
$$

where

$$
\sigma^{2}\left(\varphi_{\mathrm{A}}\right)=\sigma_{0}^{2}\left(\frac{\cos ^{2} \alpha}{\cos ^{2} \varphi_{\mathrm{A}}}+\sin ^{2} \alpha\right),
$$

where $\bar{d}$ is the mean thickness of the film, $\sigma_{0}$ denotes the rms value of the local thicknesses for normal incidence and $\alpha$ represents the angle between the thickness gradient direction 
and the plane of incidence. Note that this distribution of the local thicknesses is frequently called the Wigner semicircle distribution in the literature.

The associated ellipsometric parameters $I_{\mathrm{n}}, I_{\mathrm{S}}$ and $I_{\mathrm{C}}$ measured using phase modulated ellipsometry are the elements of the normalized Mueller matrix (see e.g., [70,77-82]). For reflected light these ellipsometric parameters are expressed by the following matrix:

$$
\hat{M}=R_{0}\left(\begin{array}{cccc}
1 & -I_{\mathrm{n}} & 0 & 0 \\
-I_{\mathrm{n}} & 1 & 0 & 0 \\
0 & 0 & I_{\mathrm{c}} & I_{\mathrm{s}} \\
0 & 0 & -I_{\mathrm{S}} & I_{\mathrm{c}}
\end{array}\right)
$$

where $R_{0}=\frac{1}{2}\left(\left\langle R_{\mathrm{p}}\right\rangle+\left\langle R_{\mathrm{S}}\right\rangle\right)$ is the total reflectance of the samples. The degree of polarization $P$ describing the depolarization of light reflected from the samples is given using these ellipsometric parameters as

$$
P=\sqrt{I_{\mathrm{s}}^{2}+I_{\mathrm{c}}^{2}+I_{\mathrm{n}}^{2}}
$$

The measured associated ellipsometric parameters of the thin films are expressed by means of the reflection coefficients of these films as follows (see e.g., $[68,77]$ ):

$$
I_{\mathrm{s}}=\mathrm{i} \frac{\left\langle r_{\mathrm{p}} r_{\mathrm{s}}^{*}\right\rangle-\left\langle r_{\mathrm{p}}^{*} r_{\mathrm{s}}\right\rangle}{\left\langle\left|r_{\mathrm{s}}\right|^{2}\right\rangle+\left\langle\left|r_{\mathrm{p}}\right|^{2}\right\rangle}, \quad I_{\mathrm{c}}=-\frac{\left\langle r_{\mathrm{p}} r_{\mathrm{s}}^{*}\right\rangle+\left\langle r_{\mathrm{p}}^{*} r_{\mathrm{s}}\right\rangle}{\left\langle\left|r_{\mathrm{s}}\right|^{2}\right\rangle+\left\langle\left|r_{\mathrm{p}}\right|^{2}\right\rangle}, \quad I_{\mathrm{n}}=\frac{\left\langle\left|r_{\mathrm{s}}\right|^{2}\right\rangle-\left\langle\left|r_{\mathrm{p}}\right|^{2}\right\rangle}{\left\langle\left|r_{\mathrm{s}}\right|^{2}\right\rangle+\left\langle\left|r_{\mathrm{p}}\right|^{2}\right\rangle}
$$

where the angle brackets again denote the mean values of the quantities, i.e.,

$$
\begin{array}{rlrl}
\left\langle r_{\mathrm{p}} r_{\mathrm{s}}^{*}\right\rangle & =\int r_{\mathrm{p}}(d) r_{\mathrm{s}}^{*}(d) \varrho(d) \mathrm{d} d, & & \left\langle r_{\mathrm{p}}^{*} r_{\mathrm{s}}\right\rangle=\int r_{\mathrm{p}}^{*}(d) r_{\mathrm{s}}(d) \varrho(d) \mathrm{d} d, \\
\left\langle\left|r_{\mathrm{s}}\right|^{2}\right\rangle=\int\left|r_{\mathrm{s}}(d)\right|^{2} \varrho(d) \mathrm{d} d, & \left\langle\left|r_{\mathrm{p}}\right|^{2}\right\rangle=\int\left|r_{\mathrm{p}}(d)\right|^{2} \varrho(d) \mathrm{d} d .
\end{array}
$$

The form of this distribution enables the efficient evaluation of the integrals in the foregoing equations by means of the numerical method of the Gaussian quadrature $[54,83]$. It should be noted that this wedge-shaped nonuniformity in thickness occurs often in practice. If the thickness nonuniformity is complicated, i.e., this nonuniformity differs from the wedge-shaped nonuniformity (see Figure 1), the other mathematical approach has to be employed. The Mueller matrix of the thin films having the thickness nonuniformity $\hat{M}$ is expressed in this way:

$$
\hat{M}=\frac{1}{S} \iint_{S} \hat{M}(d(x, y)) \mathrm{d} S,
$$

where the integration is performed over the light spot on the sample, $S$ denotes the area of this spot and $\hat{M}(d(x, y))$ is the Mueller matrix corresponding to the point on the sample with the local thickness $d(x, y)$. Note that the foregoing equation is true if the illumination by an incident light beam is constant over the entire light spot. The local thickness values can be expressed in the following way:

$$
d(x, y)=d_{0}+d_{x} \frac{x}{R}+d_{y} \frac{y}{R}+d_{x x} \frac{x^{2}}{R^{2}}+d_{x y} \frac{x y}{R^{2}}+d_{y y} \frac{y^{2}}{R^{2}}+\cdots,
$$

where $d_{0}, d_{x}, d_{y}, d_{x x}, d_{x y}, d_{y y}$, etc. are parameters of the model of thickness nonuniformity. The number of terms in the foregoing polynomial depends on the forms of the surfaces of the upper boundaries of the films. The normalization owing to a beam radius $R$ of incident light is utilized because the value of this radius cannot be determined using ellipsometric measurements. The associated ellipsometric parameters are then calculated using the following mean values: 


$$
\begin{array}{rlrl}
\left\langle r_{\mathrm{p}} r_{\mathrm{s}}^{*}\right\rangle & =\frac{1}{S} \iint_{S} r_{\mathrm{p}}(d(x, y)) r_{\mathrm{s}}^{*}(d(x, y)) \mathrm{d} S, & \left\langle r_{\mathrm{p}}^{*} r_{\mathrm{s}}\right\rangle & =\frac{1}{S} \iint_{S} r_{\mathrm{p}}^{*}(d(x, y)) r_{\mathrm{s}}(d(x, y)) \mathrm{d} S, \\
\left\langle\left|r_{\mathrm{s}}\right|^{2}\right\rangle=\frac{1}{S} \iint_{S}\left|r_{\mathrm{s}}(d(x, y))\right|^{2} \mathrm{~d} S, & \left\langle\left|r_{\mathrm{p}}\right|^{2}\right\rangle=\frac{1}{S} \iint_{S}\left|r_{\mathrm{p}}(d(x, y))\right|^{2} \mathrm{~d} S .
\end{array}
$$

An efficient method for calculating these mean values using the Gaussian quadrature and the Golub-Welsch algorithm is described in [54].

The refractive indices inside the inhomogeneous thin films with thickness nonuniformity have miscellaneous courses, i.e., they are represented by miscellaneous continuous functions of coordinate $\mathrm{z}$. In practice one can distinguish two basic groups of the profiles. The first group consists of the profile models whose complex refractive index values are the same along the upper boundaries, i.e., $n_{\mathrm{U}}$ are constant over the entire illuminated spots on samples. In other words, the optical constants at the upper boundaries do not depend on local thicknesses. For instance, the profile described by means of the following equation for the parameters $p_{\alpha}(z)$ of the dispersion model can serve as the example of these profiles:

$$
p_{\alpha}(z)=p_{\alpha}^{\mathrm{L}}+\left(p_{\alpha}^{\mathrm{U}}-p_{\alpha}^{\mathrm{L}}\right)\left(\frac{z}{d(x, y)}\right)^{s},
$$

where symbols $p_{\alpha}^{\mathrm{U}}$ and $p_{\alpha}^{\mathrm{L}}$ denote the corresponding dispersion parameters at the upper and lower boundaries. Index $\alpha$ specifies individual dispersion parameters. Symbol $s$ denotes the model parameter. The latter group is formed by the models for which the refractive index values are different along the upper boundaries. For instance, the refractive index profile described by the dispersion parameters obeying the following equation represents such example:

$$
p_{\alpha}(z)=p_{\alpha}^{\mathrm{L}}+\left(p_{\alpha}^{\mathrm{U}}-p_{\alpha}^{\mathrm{L}}\right)\left(\frac{z}{d_{0}}\right)^{s},
$$

In this case the optical constants at the upper boundaries depend on the local thicknesses.

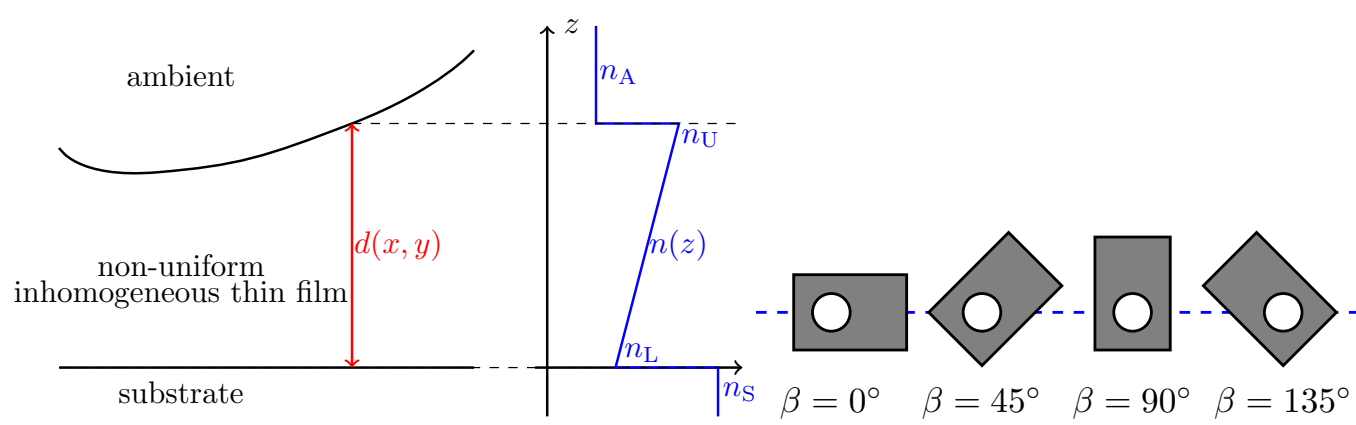

Figure 1. Schematic diagram of the inhomogeneous thin film nonuniform in thickness (left). Schematic diagram of the positions of the samples during ellipsometric measurements (right), the dashed line represents the intersection of the plane of incidence with the sample plane.

\subsubsection{Random Roughness of Film Boundaries}

Random roughness of boundaries of the homogeneous and inhomogeneous thin films is also a frequent defect. A schematic diagram of an inhomogeneous thin film with rough boundaries is shown in Figure 2.

In the literature several models for thin films with randomly rough boundaries have been presented so far. A mathematical description of these models is mainly dependent on the distribution of spatial frequencies corresponding to this random roughness. 


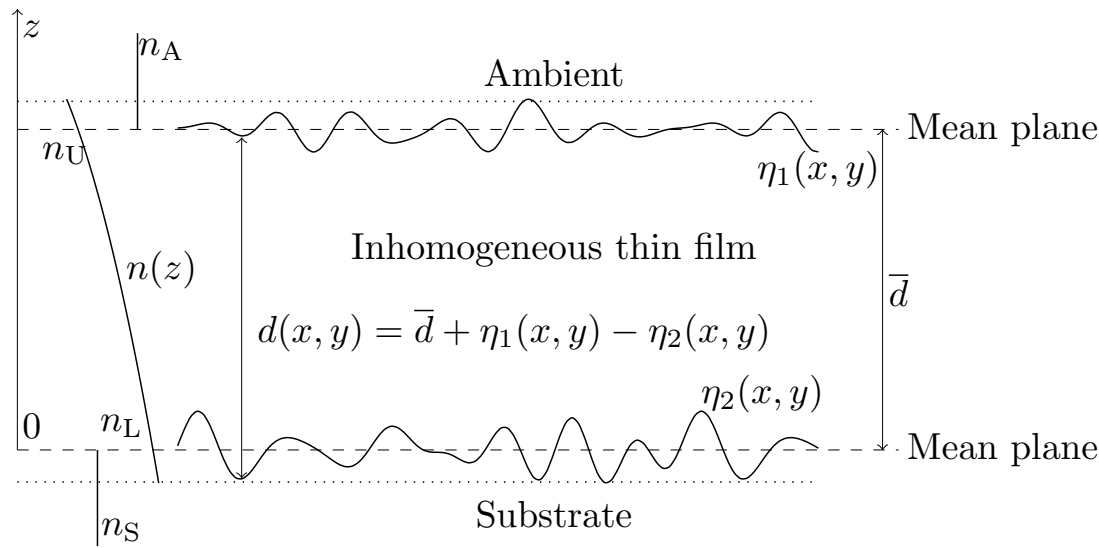

Figure 2. Schematic diagram of the inhomogeneous thin film with rough boundaries.

Random roughness exhibiting low spatial frequencies is called locally smooth roughness because the corresponding surfaces can be approximated by tangent planes in all the points of these surfaces. The scalar diffraction theory (SDT) was used to describe an interaction of light with homogeneous thin films exhibiting this random roughness (for details see e.g., [35-40,84]). In this paper it is assumed that random roughness is generated by a stationary stochastic process. Thus, it is assumed that random roughness is homogeneous and isotropic. Below we deal with coherently reflected light from inhomogeneous thin films. This coherently reflected light corresponds to the specular direction; the scattered light will not be discussed in this work. Moreover, it will be assumed that slopes of roughness are sufficiently small so that they can be neglected within the SDT. The formula for the reflection coefficients of the inhomogeneous thin films $r$ expressed by Equation (8) can then be utilized in application of the SDT. Of course, the upper and lower bounds in the integrals occurring in Equation (8) must be replaced by random functions describing roughness of the upper and lower boundaries. For instance, the following equations must be written

$$
x_{d}=\frac{4 \pi}{\lambda} \int_{\eta_{2}}^{\bar{d}+\eta_{1}} \sqrt{n^{2}(z)-n_{\mathrm{A}}^{2} \sin ^{2} \varphi_{\mathrm{A}}} \mathrm{d} z, \quad j_{1}^{(1)}=\int_{\eta_{2}}^{\bar{d}+\eta_{1}} f\left(z_{1}\right) \mathrm{e}^{-\mathrm{i} x\left(z_{1}\right)} \mathrm{d} z_{1},
$$

etc. where $\bar{d}, \eta_{1}(x, y)$ and $\eta_{2}(x, y)$ are the mean thickness, random function describing the upper boundary and random function describing the lower boundary, respectively. Then the mean values of the reflection coefficients of the rough inhomogeneous thin films $\langle r\rangle$ are given within the SDT as

$$
r_{\mathrm{SDT}}=\langle r\rangle=\int_{-\infty}^{\infty} \int_{-\infty}^{\infty} r\left(\eta_{1}, \eta_{2}\right) w\left(\eta_{1}, \eta_{2}\right) \mathrm{d} \eta_{1} \mathrm{~d} \eta_{2}
$$

where $\eta_{1} \equiv \eta_{1}(x, y), \eta_{2} \equiv \eta_{2}(x, y)$ and $w\left(\eta_{1}, \eta_{2}\right)$ is the two-dimensional distribution of probability density of the random variables $\eta_{1}$ and $\eta_{2}$. Symbol $r\left(\eta_{1}, \eta_{2}\right)$ denotes the local reflection coefficients given by Equation (8) having the integrals whose bounds contain the variables $\eta_{1}$ and $\eta_{2}$ (see Equation (36)). An example of the two-dimensional distribution function is the Gaussian (normal) distribution

$$
w\left(\eta_{1}, \eta_{2}\right)=\frac{1}{2 \pi \sigma_{1} \sigma_{2} \sqrt{1-C_{1,2}^{2}}} \exp \left[-\frac{1}{2\left(1-C_{1,2}^{2}\right)}\left(\frac{\eta_{1}^{2}}{\sigma_{1}^{2}}-2 C_{1,2} \frac{\eta_{1} \eta_{2}}{\sigma_{1} \sigma_{2}}+\frac{\eta_{2}^{2}}{\sigma_{2}^{2}}\right)\right],
$$

where $C_{1,2}$ is a cross-correlation coefficient between roughness of the upper and lower boundaries and $\sigma_{1}$ and $\sigma_{2}$ are the rms values of $\eta_{1}$ and $\eta_{2}$. In the foregoing equation it 
is assumed that the mean values of the random variables $\eta_{1}$ and $\eta_{2}$ are equal to zero. The measured reflectances and ellipsometric parameters are expressed as follows:

$$
\begin{aligned}
R_{q}=\left\langle r_{q}\right\rangle\left\langle r_{q}^{*}\right\rangle, & I_{\mathrm{s}}=\mathrm{i} \frac{\left\langle r_{\mathrm{p}}\right\rangle\left\langle r_{\mathrm{s}}^{*}\right\rangle-\left\langle r_{\mathrm{p}}^{*}\right\rangle\left\langle r_{\mathrm{s}}\right\rangle}{\left\langle r_{\mathrm{s}}\right\rangle\left\langle r_{\mathrm{s}}^{*}\right\rangle+\left\langle r_{\mathrm{p}}^{*}\right\rangle\left\langle r_{\mathrm{p}}\right\rangle}, \\
I_{\mathrm{c}}=-\frac{\left\langle r_{\mathrm{p}}\right\rangle\left\langle r_{\mathrm{s}}^{*}\right\rangle+\left\langle r_{\mathrm{p}}^{*}\right\rangle\left\langle r_{\mathrm{s}}\right\rangle}{\left\langle r_{\mathrm{s}}\right\rangle\left\langle r_{\mathrm{s}}^{*}\right\rangle+\left\langle r_{\mathrm{p}}^{*}\right\rangle\left\langle r_{\mathrm{p}}\right\rangle}, & I_{\mathrm{n}}=\frac{\left\langle r_{\mathrm{s}}\right\rangle\left\langle r_{\mathrm{s}}^{*}\right\rangle-\left\langle r_{\mathrm{p}}^{*}\right\rangle\left\langle r_{\mathrm{p}}\right\rangle}{\left\langle r_{\mathrm{s}}\right\rangle\left\langle r_{\mathrm{s}}^{*}\right\rangle+\left\langle r_{\mathrm{p}}^{*}\right\rangle\left\langle r_{\mathrm{p}}\right\rangle} .
\end{aligned}
$$

If boundary roughness exhibits high and moderate spatial frequencies, then the reflection coefficients of the inhomogeneous thin films can be calculated using the Rayleigh-Rice theory (RRT). The second-order RRT perturbation theory was applied to the homogeneous thin films and their systems in many papers (see e.g., [70,84-89]). The RRT can only be utilized if roughness has relatively small heights and slopes. Strictly speaking, these inequalities must be fulfilled: $\sigma \ll \lambda$ and $\tan \beta_{0} \lesssim 0.1$ ( $\tan \beta_{0}$ is the rms value of the slopes of roughness). The reflection coefficients $r_{\text {RRT }}$ of the inhomogeneous thin films exhibiting roughness of both the boundaries can be calculated using the approximate method described in [90]. In this approach, the reflection coefficients are expressed as $r_{\mathrm{RRT}}=r\left(\sigma_{1}, \sigma_{2}, T_{1}, T_{2}\right)$, where $r\left(\sigma_{1}, \sigma_{2}, T_{1}, T_{2}\right)$ is given by Formula (8) with the change consisting in expressing reflection coefficients of the upper and lower boundaries by means of the following equation:

$$
r_{w}=r_{0, w}+\Delta r_{w},
$$

where $w=\mathrm{U}$ and L. Symbols $\sigma_{1}, T_{1}$ and $\sigma_{2}, T_{2}$ denote the rms values of the heights and autocorrelation lengths of the upper and lower boundaries, respectively, symbol $r_{0, w}$ represents the reflection coefficients of the smooth upper and lower boundaries and symbol $\Delta r_{w}$ denotes the corrections of the second order expressed in this way:

$$
\Delta r_{w}=\int_{-\infty}^{\infty} \int_{-\infty}^{\infty} f_{w}\left(K_{x}, K_{y}\right) W_{w}\left(K_{x}^{\prime}, K_{y}\right) \mathrm{d} K_{x} \mathrm{~d} K_{y},
$$

where $K_{x}$ and $K_{y}$ denote spatial frequencies of random roughness, $f_{w}\left(K_{x}, K_{y}\right)$ represents complicated functions of $K_{x}, K_{y}, \lambda, \varphi_{\mathrm{A}}$ and optical constants of media forming the inhomogeneous thin films. Symbol $W_{\mathrm{w}}\left(K_{x}^{\prime}, K_{y}\right)$ denotes the power spectral densities functions (PSDF) of random roughness of the upper and lower boundaries. It holds that $K_{x}^{\prime}=K_{x}-(2 \pi / \lambda) n_{\mathrm{A}} \sin \varphi_{\mathrm{A}}$. The following Gaussian PSDF is often utilized in optical studies of randomly rough surfaces and thin films:

$$
W\left(K_{x}^{\prime}, K_{y}\right)=\frac{\sigma^{2} T^{2}}{4 \pi} \mathrm{e}^{-\frac{T^{2}}{4}\left(K_{x}^{\prime 2}+K_{y}^{2}\right)} .
$$

If the RRT is employed, the refractive index must be constant within the range of roughness. Furthermore, it necessary to point out that a correlation between roughness of the upper and lower boundaries is not taken into account. Therefore, the approximation expressed by Formulas (40) and (41) is usable if the films are sufficiently thick (thickness values must be in the order of hundreds of nanometers and more).

A very efficient method for calculating the optical quantities of multilayer systems comprising homogeneous thin films with randomly rough boundaries using the exact approach of the second-order perturbation RRT, i.e., using the approach taking into account the correlation among the rough boundaries, was published in [89]. This approach can easily be modified to derive the formulae for the optical quantities of the rough inhomogeneous thin films using the approximation based on the multilayer systems.

In practice we can encounter the rough thin films with roughness exhibiting wide intervals of the spatial frequencies. Then it is necessary to take into account the low, moderate and high spatial frequencies in expressing the reflection coefficients of thin films. In this case one can utilize the approximation based on the formulae for the reflection 
coefficients $\langle r\rangle$ (see Equation (37)). In this equation the reflection coefficients of the upper and lower boundaries must be expressed by means of Formula (40). Thus, the reflection coefficients $r_{\mathrm{SDT}+\mathrm{RRT}}$ of the inhomogeneous thin films exhibiting this roughness are based on the heuristic combination of the SDT and RRT. Of course, the mathematical calculations of such the reflection coefficients using numerical procedures are very difficult. The same approximation can be used to calculate of the reflection coefficients of the inhomogeneous thin films with composite random roughness (composite roughness contains, for example, low and high spatial frequencies). The coefficients $r_{\mathrm{RRT}}$ and $r_{\mathrm{SDT}+\mathrm{RRT}}$ can be employed for calculating the optical quantities in the same way as coefficients $r_{\mathrm{SDT}}$ (see Equation (39)). Note that this combination of the SDT and RRT was utilized for calculating the ellipsometric parameters and reflectance of randomly rough surfaces of $\mathrm{Si}$ and GaAs covered by overlayers (see $[46,91])$.

Some inhomogeneous thin films exhibit constant complex refractive indices within regions that are more extended than regions occupied by roughness of the upper and lower boundaries. This means that the homogeneous thin films adjacent to these rough boundaries must be considered. The smooth inhomogeneous thin films occurring between these two rough homogeneous films are simultaneously taken into account. It is assumed that roughness contains the low spatial frequencies, i.e,. this roughness corresponds to the SDT. It is again assumed that slopes of this roughness are negligible. The inhomogeneous thin films with smooth boundaries occurring between the two rough homogeneous films can be represented by effective boundaries whose reflection and transition coefficients are identical with those presented for the inhomogeneous thin films in [76]. The formula for the reflection coefficients of this model of the inhomogeneous film can be derived using the formula for the double-layer system containing two homogeneous thin films and three randomly rough boundaries presented in [92]. If the three-dimensional Gaussian distribution of the heights of roughness is utilized then the following formula for the reflection coefficients $r_{3,2}$ of this system is obtained:

$$
r_{3,2}=\langle r\rangle_{0}+\langle r\rangle_{1}+\langle r\rangle_{2}
$$

where

$$
\begin{aligned}
& \langle r\rangle_{0}=r_{1} \mathrm{e}^{-\frac{1}{2} E_{0}^{2} S_{1,1}}, \\
& \langle r\rangle_{1}=\sum_{p=1}^{\infty} \mathrm{e}^{\mathrm{i} 2 p \bar{x}_{1}} Q_{1}(p) H_{1}(p), \\
& \langle r\rangle_{2}=\sum_{p=2}^{\infty} \sum_{m_{1}=1}^{p-1} \mathrm{e}^{\mathrm{i} 2 m_{1} \bar{x}_{1}+2 \mathrm{i}\left(p-m_{1}\right) \bar{x}_{2}} Q_{2}\left(m_{1}, p-m_{1}\right) H_{2}\left(m_{1}, p-m_{1}\right),
\end{aligned}
$$

where

$$
\begin{aligned}
Q_{1}(p) & =t_{1} t_{1}^{\prime}\left(r_{1}^{\prime}\right)^{p-1} r_{2}^{p} \\
H_{1}(p) & =\mathrm{e}^{-\frac{1}{2} D_{1}^{2} S_{1,1}-\frac{1}{2} D_{2}^{2} S_{2,2}-D_{1} D_{2} S_{1,2},} \\
Q_{2}\left(m_{1}, m_{2}\right) & =t_{1} t_{1}^{\prime}\left(r_{1}^{\prime}\right)^{m_{1}-1} r_{3}^{m_{2}} \sum_{v_{2}=1}^{\min \left(m_{1}, m_{2}\right)}\left(\begin{array}{c}
m_{1} \\
v_{2}
\end{array}\right)\left(\begin{array}{c}
m_{2}-1 \\
v_{2}-1
\end{array}\right) r_{2}^{m_{1}-v_{2}}\left(t_{2} t_{2}^{\prime}\right)^{v_{2}}\left(r_{2}^{\prime}\right)^{\left(m_{2}-v_{2}\right)}, \\
H_{2}\left(m_{1}, m_{2}\right) & =\mathrm{e}^{-\frac{1}{2} D_{1}^{2} S_{1,1}-\frac{1}{2} D_{2}^{2} S_{2,2}-\frac{1}{2} D_{3}^{2} S_{3,3}-D_{1} D_{2} S_{1,2}-D_{1} D_{3} S_{1,3}-D_{2} D_{3} S_{2,3},}
\end{aligned}
$$

where $m_{1}$ and $m_{2}$ express how many times light passes through the upper and lower thin films, respectively, $p=m_{1}+m_{2}, \bar{x}_{i}=\frac{4 \pi}{\lambda} n_{i} \bar{d}_{i} \cos \varphi_{i}(i=1,2), n_{1}, \bar{d}_{1}, \varphi_{1}$ and $n_{2}, \bar{d}_{2}, \varphi_{2}$ are the refractive index, mean thicknesses and refraction angle of the upper and lower thin film, respectively, $D_{j}=E_{j}-E_{j-1}, E_{j}=\frac{4 \pi}{\lambda} m_{j} n_{j} \cos \varphi_{j},(j=0,1,2), m_{0}=1$ and $m_{j}=0$ for $j>g\left(g=0\right.$ for $\langle r\rangle_{0}, g=1$ for $\langle r\rangle_{1}$, and $g=2$ for $\left.\langle r\rangle_{2}\right)$. Thus, $E_{0}=\frac{4 \pi}{\lambda} n_{\mathrm{A}} \cos \varphi_{\mathrm{A}}$, $D_{1}=E_{1}-E_{0}, D_{2}=E_{2}-E_{1}, D_{3}=-E_{2}, E_{1}=\frac{4 \pi}{\lambda} m_{1} n_{1} \cos \varphi_{1}, E_{2}=\frac{4 \pi}{\lambda} m_{2} n_{2} \cos \varphi_{2}$, 
$S_{i, j}=\sigma_{i} \sigma_{j} C_{i, j},(i=1,2,3), C_{i, j}$ denotes the correlation coefficient among the boundaries, $C_{i, i}=1$. Note that the boundaries are numbered from the ambient to substrate. Symbols $r_{1}, r_{1}^{\prime}$ and $t_{1}, t_{1}^{\prime}$ denote the reflection and transmission coefficients of the first (uppermost) boundary, $r_{3}$ are the reflection coefficients of the third (lower) boundary, $r_{2}, r_{2}^{\prime}$ and $t_{2}, t_{2}^{\prime}$ are the reflection and transmission coefficients of the second (medium) boundary. Symbols without primes correspond to light falling onto the system from the ambient-side while symbols with primes correspond to light falling onto system from the substrate-side.

The formula for the reflection coefficients $\bar{r}_{\mathrm{I}}$ of the model consisting of the smooth inhomogeneous film surrounded by two rough homogeneous thin films is obtained from Equation for $r_{3,2}$ if it is put: $\sigma_{2}=0$ (see Equation (43)). Moreover, the Fresnel coefficients $r_{2}, r_{2}^{\prime}, t_{2}$ and $t_{2}^{\prime}$ must be replaced by the corresponding coefficients for the inhomogeneous films given by Equation (8)

Formula (43) was derived using the general formula for the reflection coefficients of the multilayer system containing homogeneous thin films with randomly rough boundaries. This derivation is based on multiple beam interference model different from that utilized for expressing inhomogeneity (for details see [93]).

The formula for the reflection coefficients of the inhomogeneous thin films with randomly rough boundaries is obtained from the formula for $\bar{r}_{\mathrm{I}}$ by using $x_{1}=x_{2}=0$, $r_{1}=r_{\mathrm{U}}$ and $r_{3}=r_{\mathrm{L}}$. Of course, it must be fulfilled that the refractive indices and extinction coefficients are constant within the range of random roughness of both the boundaries.

If the inhomogeneous thin films exhibit only one rough boundary, the formula for the reflection coefficients $\bar{r}_{\text {SDT }}$ of this film is easily derived using equation $\bar{r}_{\text {SDT }}=\langle r\rangle_{0}+\langle r\rangle_{1}$ in which it is taken into account that $r_{1}=r_{\mathrm{U}}$ and $r_{2}=\tilde{r}$, where $\tilde{r}$ is given by (8). However, in the Equation (8) it must be set $n_{\mathrm{A}}=n_{\mathrm{U}}$. Thus, one can write:

$$
\bar{r}_{\mathrm{SDT}}=r_{\mathrm{U}} \chi\left(v_{z}\right)+t_{\mathrm{U}} t_{\mathrm{U}}^{\prime} \sum_{m=0}^{\infty} r_{\mathrm{U}}^{m} \tilde{r}^{(m+1)} \chi\left(B_{m}\right)
$$

where $v_{z}=(4 \pi / \lambda) n_{\mathrm{A}} \cos \varphi_{\mathrm{A}}, B_{m}=v_{z}+(4 \pi / \lambda)(m+1) n_{\mathrm{U}} \cos \varphi_{\mathrm{U}}$ and $\chi(\mu)=$ $\exp \left(-\frac{1}{2} \sigma_{1}^{2} \mu^{2}\right)$. It holds, that $\mu=v_{z}$ or $B_{m}$. The similar formula can be derived for the inhomogeneous thin films with the rough lower boundaries and smooth upper boundaries.

An influence of slopes of roughness on the optical quantities of the inhomogeneous thin films has not been studied in the SDT so far. This is probably given by considerable difficulties connected with a theoretical solution of this problem. Fortunately, the most inhomogeneous thin films exhibiting random roughness with negligible slopes are encountered in the optical characterization of thin films in practice.

\subsubsection{Uniaxial Anisotropy}

An internal stress often occurs inside many homogeneous and inhomogeneous thin films. This can be caused by various reasons. For example, it is caused by misfits between crystallographic structures of these films and their substrates. The stress can generate artificial optical anisotropy inside the films even when materials forming these films are optically isotropic. This uniaxial anisotropy with the optical axis perpendicular to the parallel boundaries is frequently exhibited by the thin films. In this paper we will therefore deal with the influence of this kind of optical anisotropy on the optical quantities of the inhomogeneous thin films in reflected light. The inhomogeneous thin films with this defect are described by the complex dielectric tensor, i.e.,

$$
\varepsilon(z)=\left(\begin{array}{ccc}
\varepsilon_{\mathrm{o}}(z) & 0 & 0 \\
0 & \varepsilon_{\mathrm{o}}(z) & 0 \\
0 & 0 & \varepsilon_{\mathrm{e}}(z)
\end{array}\right)
$$

where $\varepsilon_{\mathrm{o}}(z)$ and $\varepsilon_{\mathrm{e}}(z)$ are the ordinary and extraordinary complex dielectric functions, respectively. The complex refractive indices $n_{\mathrm{o}}(z)$ and $n_{\mathrm{e}}(z)$ are expressed as $n_{\mathrm{o}}(z)=\left(\varepsilon_{\mathrm{o}}(z)\right)^{1 / 2}$ and $n_{\mathrm{e}}(z)=\left(\varepsilon_{\mathrm{e}}(z)\right)^{1 / 2}$. 
For including this defect into formulae for the reflection coefficients of the inhomogeneous thin films the approximation based on the multilayer systems is used (see Section 2.1.2). Thus, the inhomogeneous film is divided by $N$ boundaries into $N+1$ layers in the way depicted in Figure 3. The thickness values of the inner layers are $H=d / N$, where $d$ is the total thickness of the film. Note that the first and the last layer have half the thickness of the inner layers. It should be emphasized that the orientation and origin of the $z$ axis used in this section is different from that chosen is the previous sections. The ordinary and extraordinary dielectric functions of the individual layers are as follows:

$$
\varepsilon_{\mathrm{o}, k}=\varepsilon_{\mathrm{o}}\left(z_{k}\right), \quad \varepsilon_{\mathrm{e}, k}=\varepsilon_{\mathrm{e}}\left(z_{k}\right), \quad z_{k}=k \frac{d}{N}, \quad k=0,1,2, \ldots, N .
$$

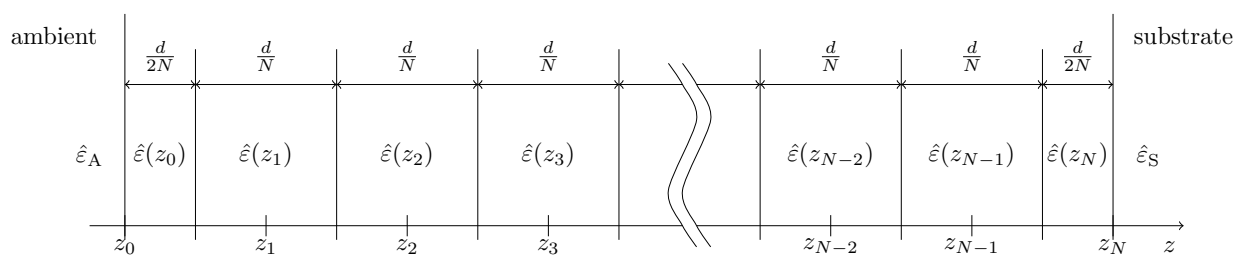

Figure 3. Schematic diagram of the system of thin homogeneous layers approximating the inhomogeneous layer.

The reflection coefficients of the multilayer systems can be calculated using the Yeh matrix formalism [94]. It should be noted that other matrix formalisms can be used to solve this special type of anisotropy considered here $[73,77]$. If the plane of incidence is identical with the coordinate plane $(x, z)$ then the $y$-components of the wavevectors vanish and the $\mathrm{x}$-components are given as $k_{x}=k_{0} \sin \varphi_{\mathrm{A}}$, where $k_{0}=2 \pi n_{\mathrm{A}} / \lambda$. The z-components of the wavevectors of the ordinary and extraordinary waves inside the $k$-th medium are equal to $\pm k_{\mathrm{o} z, k}$ and $\pm k_{\mathrm{e} z, k}$ expressed as follows (see $[67,77,94]$ ):

$$
k_{\mathrm{o} z, k}=k_{0} \sqrt{\varepsilon_{\mathrm{o}, k}-n_{\mathrm{A}}^{2} \sin ^{2} \varphi_{\mathrm{A}}}, \quad k_{\mathrm{e} z, k}=k_{0} \sqrt{\frac{\varepsilon_{\mathrm{o}, k}}{\varepsilon_{\mathrm{e}, k}}\left(\varepsilon_{\mathrm{e}, k}-n_{\mathrm{A}}^{2} \sin ^{2} \varphi_{\mathrm{A}}\right)},
$$

In the Yeh matrix formalism the multilayer system is described using the boundary and phase matrices. The boundary matrices express reflection and refraction of the waves at the boundaries between adjacent media. The phase matrices express changes of wave phases at propagating these waves through layers. The boundary matrix describing the boundary between the $(k-1)$-th and $k$-th media is given as

$$
\hat{B}_{k-1, k}=\left(\begin{array}{cccc}
B_{k-1, k}^{(1)} & B_{k-1, k}^{(2)} & 0 & 0 \\
B_{k-1, k}^{(2)} & B_{k-1, k}^{(1)} & 0 & 0 \\
0 & 0 & B_{k-1, k}^{(3)} & B_{k-1, k}^{(4)} \\
0 & 0 & B_{k-1, k}^{(4)} & B_{k-1, k}^{(3)}
\end{array}\right)
$$

where the elements of this matrix are given in this way:

$$
\begin{aligned}
B_{k-1, k}^{(1)} & =\frac{1}{2} \frac{\alpha_{k}}{\alpha_{k-1}}\left(\frac{Y_{\mathrm{e}, k}}{Y_{\mathrm{e}, k-1}}+1\right), & B_{k-1, k}^{(2)} & =\frac{1}{2} \frac{\alpha_{k}}{\alpha_{k-1}}\left(\frac{Y_{\mathrm{e}, k}}{Y_{\mathrm{e}, k-1}}-1\right), \\
B_{k-1, k}^{(3)} & =\frac{1}{2}\left(\frac{Y_{\mathrm{o}, k}}{Y_{\mathrm{o}, k-1}}+1\right), & B_{k-1, k}^{(4)} & =\frac{1}{2}\left(\frac{Y_{\mathrm{o}, k}}{Y_{\mathrm{o}, k-1}}-1\right) .
\end{aligned}
$$

The admittances for ordinary and extraordinary waves and quantity $\hat{\alpha}_{k}$ are expressed as 


$$
Y_{\mathrm{o}, k}=\frac{k_{\mathrm{o}, z, k}}{k_{0}}, \quad Y_{\mathrm{e}, k}=\frac{k_{0} \varepsilon_{\mathrm{o}, k}}{k_{\mathrm{e}, z, k}}, \quad \alpha_{k}=\frac{k_{\mathrm{e}, z, k}}{\sqrt{\frac{k_{0}^{2} \varepsilon_{\mathrm{o}, k}^{2}}{\varepsilon_{\mathrm{e}, k}}-\left(\frac{\varepsilon_{\mathrm{o}, k}}{\varepsilon_{\mathrm{e}, k}}-1\right) k_{\mathrm{e}, z, k}^{2}}}
$$

The propagation of the waves inside the layer with thickness $H$ corresponding to the $k$-th medium is described by the phase matrix as

$$
\hat{T}_{k}(H)=\left(\begin{array}{cccc}
\mathrm{e}^{-\mathrm{i} H k_{\mathrm{e}, z, k}} & 0 & 0 & 0 \\
0 & \mathrm{e}^{\mathrm{i} H k_{\mathrm{e}, z, k}} & 0 & 0 \\
0 & 0 & \mathrm{e}^{-\mathrm{i} H k_{\mathrm{o}, z, k}} & 0 \\
0 & 0 & 0 & \mathrm{e}^{\mathrm{i} H k_{\mathrm{o}, z, k}}
\end{array}\right)
$$

The transfer matrix of the multilayer system $\hat{S}$ approximating the inhomogeneous thin film is calculated as a product of the boundary and phase matrices, i.e.,

$$
\hat{S}=\hat{B}_{\mathrm{A}, 0} \hat{T}_{0}(H / 2) \hat{B}_{0,1} \hat{T}_{1}(H) \hat{B}_{1,2} \hat{T}_{2}(H) \cdots \hat{B}_{N-2, N-1} \hat{T}_{N-1}(H) \hat{B}_{N-1, N} \hat{T}_{N}(H / 2) \hat{B}_{N, S},
$$

where $\hat{B}_{\mathrm{A}, 0}$ is the boundary matrix of the boundary between the ambient and the upper layer and $\hat{B}_{N, S}$ represents the boundary between the lower film ( $N$-th film) and the substrate. The reflection coefficients of the inhomogeneous thin film for the p-polarization (extraordinary waves) and s-polarization (ordinary waves) are given as follows [67]:

$$
r_{\mathrm{I}, \mathrm{p}}=\frac{S_{21}}{S_{11}}, \quad r_{\mathrm{I}, \mathrm{s}}=\frac{S_{43}}{S_{33}}
$$

where $S_{i j}$ are elements of matrix $\hat{S}$. The foregoing equations are valid for light falling onto the system from the ambient side.

To achieve exact results corresponding to these anisotropic inhomogeneous thin films it is necessary to perform the limit in which the number of approximating homogeneous layers goes to infinity. As mentioned above, it was shown that the convergence to the exact results could be improved by the Richardson extrapolation [33]. This paper dealt with the isotropic inhomogeneous films but the results presented can easily be generalized to anisotropic inhomogeneous films.

\section{Examples of Optical Characterization}

In this section the application of the theoretical results to the optical characterization of selected inhomogeneous thin films exhibiting the defects discussed above are presented. The results concerning two examples of optical characterization are presented. These examples were carefully selected such that most of the approaches discussed in the theoretical part are accompanied by examples of their utilization in the optical characterization of inhomogeneous films occurring in practice. The results achieved in the complete optical characterization of the inhomogeneous polymer-like thin film of $\mathrm{SiO}_{x} \mathrm{C}_{y} \mathrm{H}_{z}$ exhibiting the complicated thickness nonuniformity and transition layer and inhomogeneous thin film of nonstoichiometric silicon nitride $\left(\mathrm{SiN}_{x}\right)$ with random roughness of the upper boundary and uniaxial anisotropy are introduced here. The complete optical characterization of these films consists in determining all the parameters describing their optical properties. Both the thin films were deposited onto the silicon single crystal substrates. Before the deposition of the polymer-like film, the silicon surface was pretreated in argon (Ar) discharge. This resulted in a non-negligible transition layer at the silicon surface. Therefore, it is necessary to deal with the optical characterization of this layer in the first stage of the optical characterization of the $\mathrm{SiO}_{x} \mathrm{C}_{y} \mathrm{H}_{z}$ thin film. 


\subsection{Optical Characterization of the Transition Layer}

The silicon substrate was pretreated $5 \mathrm{~min}$ in Ar discharge at applied power of $50 \mathrm{~W}$. The flow of argon was of $1 \mathrm{sccm}$. The optical characterization of the transition layer was performed using variable-angle spectroscopic ellipsometry (see below). Since the measurements were realized in air it was necessary to assume an overlayer corresponding to a native oxide layer (NOL) onto upper boundary of the transition layer. The combination of the Campi-Coriasso dispersion model with two terms and the exponential tail representing weak absorption below the band gap energy together with the Kramers-Kroning relation was utilized for determining the spectral dependencies of the optical constants of this transition layer. The optical constants of the silicon substrate were fixed in the values taken from the literature [95].

In Figure 4 (left) the determined spectral dependencies of the optical constants for the characterized transition layer are plotted. These optical constants are considerably different from the optical constants of silicon single crystal but they are relatively close to the optical constants of amorphous silicon. The optical constants of amorphous silicon are taken from [96]. This implies that the material of this transition layer is closer to amorphous silicon than to crystalline silicon. It can be expected that the transition layer represents some damaged layer under the surface of crystalline silicon originated in the process of pretreating this surface in argon discharge. The thickness values of this transition layer and NOL are $d_{\mathrm{T}}=12.4 \pm 0.2 \mathrm{~nm}$ and $d_{0}=2.9 \pm 0.2 \mathrm{~nm}$, respectively. The spectral dependencies of the optical constants of the native oxide layer were fixed in those of amorphous $\mathrm{SiO}_{2}$ published in [97]. In Figure 4 (right) the spectral dependencies of the ellipsometric parameters corresponding to the system $\mathrm{Si} /$ transition layer/NOL are depicted. It is seen that the agreement between the experimental and theoretical data is good which supports a correctness of the results obtained for the transition layer and NOL. The spectral dependencies of the optical constants and thickness value of the transition layer presented in this paragraph are fixed in the optical characterization of the $\mathrm{SiO}_{x} \mathrm{C}_{y} \mathrm{H}_{z}$ thin film.
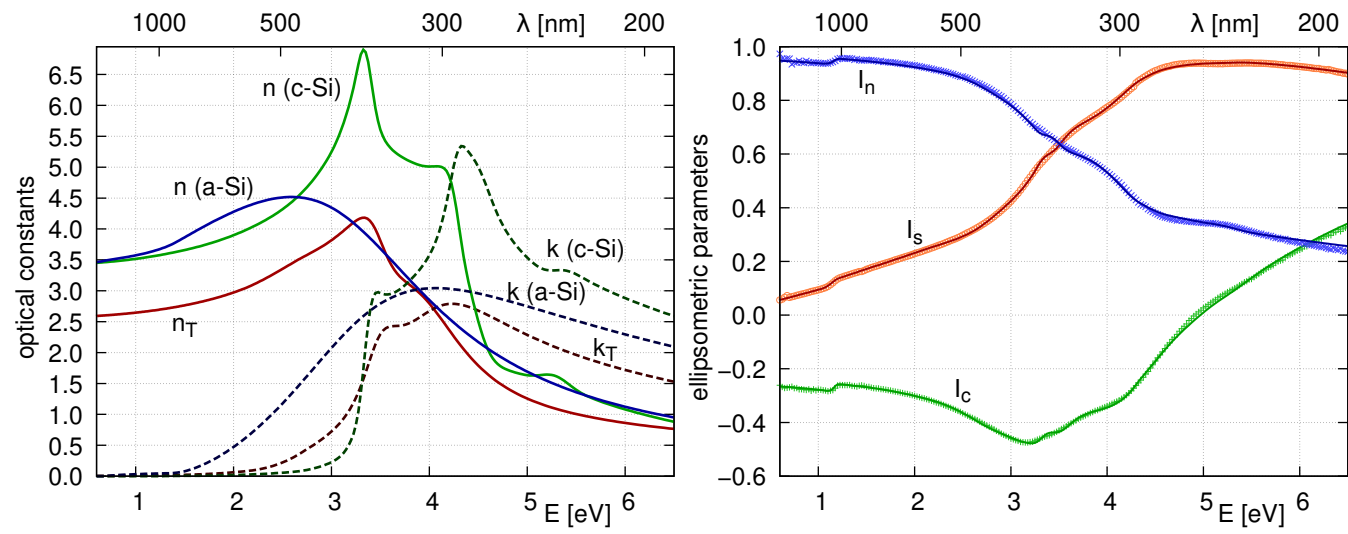

Figure 4. Spectral dependencies of the optical constants of the transition layer (left) and the associated ellipsometric parameters measured at angle of incidence $70^{\circ}$ on the sample pretreated in the argon discharge (right). The points represent the experimental values while the lines represent the theoretical values.

\subsection{Optical Characterization of the Inhomogeneous $\mathrm{SiO}_{x} \mathrm{C}_{y} \mathrm{H}_{z}$ Thin Film}

\subsubsection{Sample Preparation and Experimental Arrangement}

This film was prepared by the plasma enhanced chemical vapor deposition (PECVD) onto the silicon single crystal substrate. The deposition was performed by means of a parallel plate reactor with capacitively coupled discharge at working frequency of $13.56 \mathrm{MHz}$. The reaction chamber was made of a glass cylinder closed by two stainless flanges. The parallel electrodes were made of graphite and RF power was applied to the lower electrode (substrate). By using a blocking capacitor, negative DC self-bias voltage was induced at the 
substrate electrode to control the acceleration of ions bombarding the growing films. The mixture of methane $\left(\mathrm{CH}_{4}\right)$ and hexamethyldisiloxane $\left(\mathrm{C}_{6} \mathrm{H}_{18} \mathrm{Si}_{2} \mathrm{O}-\mathrm{HMSDO}\right)$ was supplied into the reactor chamber by the glass torus with many outlets on its perimeter. In order to vary the composition of the $\mathrm{SiO}_{x} \mathrm{C}_{y} \mathrm{H}_{z}$ thin film the methane flow rate was gradually reduced from $5.5 \mathrm{sccm}$ to $0 \mathrm{sccm}$ for $5 \mathrm{~min}$. The remaining details of preparing this film are presented in [68]. The thin film was deposited onto one side of double side polished unheated silicon substrate with temperature about $30{ }^{\circ} \mathrm{C}$. In order to create the $\mathrm{SiO}_{x} \mathrm{C}_{y} \mathrm{H}_{z}$ thin films with pronounced thickness nonuniformity the samples were positioned near the edges of the holder where the growth of the film is influenced by the distorted electric field. The structural model of this film is schematically depicted in Figure 1 (left).

A Horiba Jobin Yvon UVISEL phase modulated ellipsometer (HORIBA Jobin Yvon, Irvine, CA, USA) was used to measure the associated ellipsometric parameters. The spectral dependencies of these parameters were measured for five angles of incidence in the interval $55-75^{\circ}$ within the spectral range $0.6-6.5 \mathrm{eV}(191-2066 \mathrm{~nm})$. The ISR data were measured at normal incidence in the spectral range $1.7-4.5 \mathrm{eV}(275-700 \mathrm{~nm})$ using the original self-made imagining spectrophotometer. The pixel size was $80 \times 80 \mu \mathrm{m}$.

\subsubsection{Dispersion Model}

The Campi-Coriasso dispersion model with one term is used to express the dielectric response of the film characterized. The imaginary part of $\varepsilon_{\mathrm{i}}(E)$ of the complex dielectric function $\varepsilon(E)$ of this film is given as follows [98,99]:

$$
\varepsilon_{\mathrm{i}}(E)=\frac{2 N_{\mathrm{vc}}}{\pi E} \frac{B\left(E-E_{\mathrm{g}}\right)^{2} \Theta\left(E-E_{\mathrm{g}}\right)}{\left[\left(E_{\mathrm{c}}-E_{\mathrm{g}}\right)^{2}-\left(E-E_{\mathrm{g}}\right)^{2}\right]^{2}+B^{2}\left(E-E_{\mathrm{g}}\right)^{2}},
$$

where $E$ denotes photon energy, $N_{\mathrm{vc}}$ is the strength of the interband electronic transitions, $\theta($.$) represents the Heaviside function, E_{\mathrm{g}}$ is the band gap energy and $E_{\mathrm{c}}$ and $B$ are the parameters $\left(E_{\mathrm{c}}>E_{\mathrm{g}}\right)$.

The profile of the imaginary part of the dielectric function of the $\mathrm{SiO}_{x} \mathrm{C}_{y} \mathrm{H}_{z}$ thin film is modeled by considering the dispersion parameters as functions of coordinate $z$. It was found that the dependencies of the dispersion parameters $p_{\beta}(z)$ corresponding to Equation (34) for $s=1$ were satisfactory for the processing of the experimental data measured for this $\mathrm{SiO}_{x} \mathrm{C}_{y} \mathrm{H}_{z}$ thin film.

The spectral dependency of the real part $\varepsilon_{\mathrm{r}}(E)$ of the dielectric function $\varepsilon(E)$ of the $\mathrm{SiO}_{x} \mathrm{C}_{y} \mathrm{H}_{z}$ thin film were again calculated by means of the Kramers-Kronig relation.

\subsubsection{Data Processing}

The experimental data consisting of the measured associated ellipsometric parameters were processed by Formulas (29) and (33). The values of all the ellipsometric parameters were processed simultaneously by means of the least-squares method (LSM). The spectral dependencies of the optical constants of the silicon single crystal substrate were again fixed in values presented in [95]. It was shown that the use of the numerical method based on the Chebysev interpolation for calculating the integrals corresponding to inhomogeneity and numerical method utilizing the Gaussian quadrature for calculating integrals corresponding the thickness nonuniformity enormously increased the efficiency of processing the experimental data. The ellipsometric parameters are strongly sensitive to the shape of thickness nonuniformity along the direction lying in the plane of incidence where the light spot is the longest. This is why the ellipsometric parameters were measured for four orientations of the sample owing to the incidence plane to determine thickness nonuniformity in a more detailed way. These orientations are shown in Figure 1 (right). The characterized sample was covered by a mask with a circular hole so that selected parts of the inhomogeneous nonuniform thin film were easily identified. The measured spectral dependencies of the ellipsometric parameters were processed simultaneously for all incidence angles and all the rotations corresponding to the individual orientations. These rotations correspond to 
the azimuth angles $\beta=0^{\circ}, 45^{\circ}, 90^{\circ}$ and $135^{\circ}$ (see Figure 1). ISR was used to confirm the results concerning the thickness nonuniformity achieved by means of ellipsometry.

\subsubsection{Results and Discussion}

The polynomial expression of thickness nonuniformity is utilized for this inhomogeneous thin film of $\mathrm{SiO}_{x} \mathrm{C}_{y} \mathrm{H}_{z}$ (see Equation (32)). The determined values of the coefficients describing thickness nonuniformity of this film corresponding to the polynomial of the second order are summarized in Table 1. These values are compared with those found using ISR. Note that within the processing of the ISR data the values of the spectral dependencies of the optical constants of the $\mathrm{SiO}_{x} \mathrm{C}_{y} \mathrm{H}_{z}$ thin film were fixed in those determined by means of spectroscopic ellipsometry. Thus, the values of the local thicknesses belonging to individual pixels were the only parameters determined by ISR. On the basis of these local thicknesses the values of the coefficients of the quadratic polynomial were determined. The results presented in Table 1 imply that the differences in values of the corresponding coefficients of nonuniformity in thickness found using ellipsometry and ISR are rather small.

Table 1. Values of the parameters describing the thickness nonuniformity of the characterized film of $\mathrm{SiO}_{x} \mathrm{C}_{y} \mathrm{H}_{z}$ (left). Values of quantity $\chi$ for the individual total corrections in the formulae for the reflection coefficients of the inhomogeneous $\mathrm{SiO}_{x} \mathrm{C}_{y} \mathrm{H}_{z}$ thin film (right).

\begin{tabular}{lccccc}
\hline & & Ellipsometry & ISR & & $\chi$ \\
\hline$d_{0}$ & {$[\mathrm{~nm}]$} & $960.7 \pm 1.1$ & $959.5 \pm 0.3$ & WKBJ & 5.813 \\
$d_{\mathrm{x}}$ & {$[\mathrm{nm}]$} & $9.7 \pm 0.1$ & $14.78 \pm 0.06$ & with term 1 & 5.419 \\
$d_{\mathrm{y}}$ & {$[\mathrm{nm}]$} & $9.5 \pm 0.1$ & $11.10 \pm 0.06$ & with terms 1+2 & 5.418 \\
$d_{\mathrm{xx}}$ & {$[\mathrm{nm}]$} & $2.01 \pm 0.09$ & $2.73 \pm 0.03$ & with terms 1+2 +3 & 5.418 \\
$d_{\mathrm{xy}}$ & {$[\mathrm{nm}]$} & $1.35 \pm 0.09$ & $1.30 \pm 0.03$ & wedge & 5.807 \\
$d_{\mathrm{yy}}$ & {$[\mathrm{nm}]$} & $1.69 \pm 0.09$ & $2.23 \pm 0.03$ & & \\
\hline
\end{tabular}

In Figure 5 (left) the spectral dependencies of the optical constants corresponding to the upper and lower boundaries of this polymer-like film are depicted. From this figure it is seen that the differences between the spectral dependencies of the optical constants at the upper and lower boundary are relatively large. The profiles of the optical constants for $E=4 \mathrm{eV}$ are also shown in Figure 5 (right). The characterized film is absorbing for $E \geq 2.5 \mathrm{eV}$ in the region close to upper boundary. The quality of the fits of the experimental data is expressed using the quantity $\chi$ defined as $\chi^{2}=\Sigma / N_{\exp }$, where symbols $\Sigma$ and $N_{\exp }$ denote the residual sum of squares and number of all measured values, respectively. By means of quantity $\chi$ it was found that the reflection coefficients of this $\mathrm{SiO}_{x} \mathrm{C}_{y} \mathrm{H}_{z}$ thin film could be expressed as $r=r_{0}+\Delta r_{1}$ which implies the small gradients of the profiles of the optical constants of this film which is caused by the large values of the local thickness of the characterized film (see Table 1). If the inhomogeneous thin films exhibit the larger values of the gradients of the profiles of their optical constants then the total corrections of the higher orders must be included into the expression for the reflection coefficients. The profiles of the local thicknesses of the film determined by ellipsometry and ISR for the four orientations are plotted in Figure 6. These profiles correspond to the cross-sections of the surface of the upper boundary with the incidence plane corresponding to the rotations for angles $\beta=0^{\circ}, 90^{\circ}$ and $45^{\circ}, 135^{\circ}$. These profiles determined by ISR are also introduced for comparison in this figure. The profiles of the local thicknesses corresponding to ISR are directly evaluated from the map of the local thicknesses. The maps of the local thicknesses and three-dimensional views of the upper boundary surface determined by ellipsometry and ISR are shown in Figures 7 and 8. The results presented in Figures 6-8 imply that using ellipsometry, the surface of the upper boundary of the characterized film was determined in a correct way. The fits of the spectral dependencies of the associated ellipsometric parameters measured for incidence angle of $70^{\circ}$ and azimuth angle $\beta=0^{\circ}$ are depicted in Figure 9 (left). One can see that these fits are very good which supports a correctness of 
the results presented for this inhomogeneous nonuniform thin film. In Figure 9 (right) the spectral dependencies of the degree of polarization of this film for three incidence angles are presented. It is seen that the $\mathrm{SiO}_{x} \mathrm{C}_{y} \mathrm{H}_{z}$ film exhibits the considerable depolarization. This depolarization is mainly caused by the thickness nonuniformity. The depolarization does not occur for photon energies $E \geq 4.5 \mathrm{eV}$ because the film is not transparent in this region. The thickness nonuniformity has no influence on reflected light in this range.

From the foregoing it is evident that the method of VASE described in this paper enables us to carry out the complete optical characterization of the inhomogeneous polymerlike film exhibiting the combination of transition layer and complicated thickness nonuniformity. This can also be expected for other inhomogeneous thin films with the same defects.

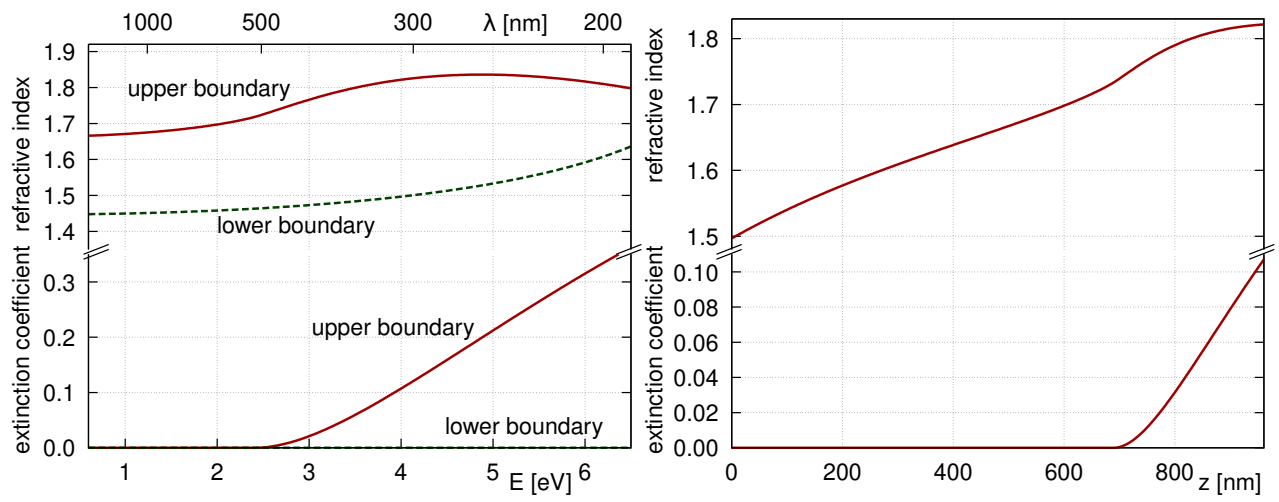

Figure 5. The spectral dependencies (left) and the depth profile at $E=4 \mathrm{eV}$ (right) of the optical constants of the inhomogeneous layer $\mathrm{SiO}_{x} \mathrm{C}_{y} \mathrm{H}_{z}$ thin film.
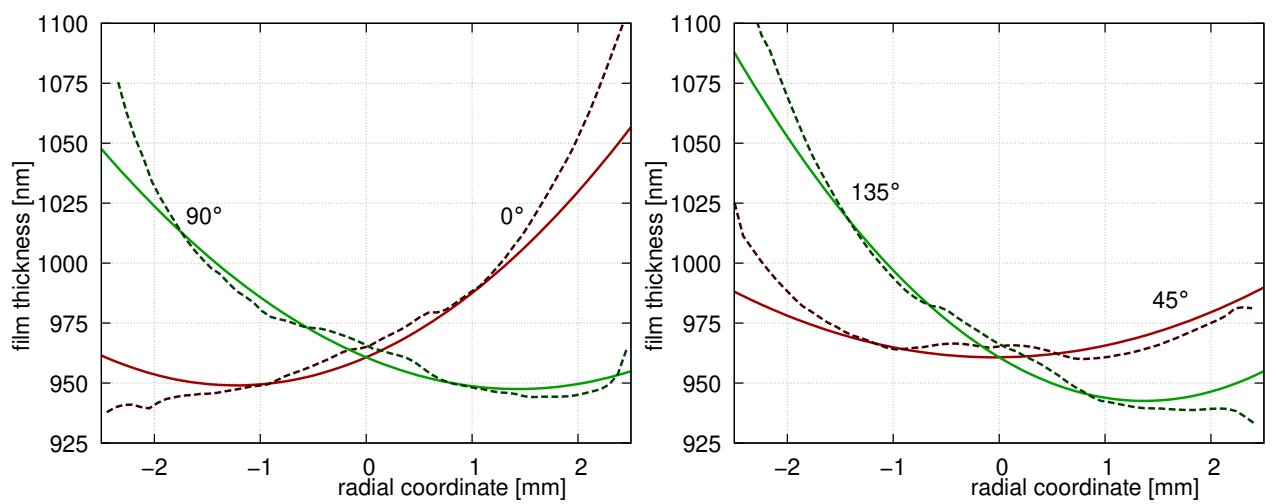

Figure 6. The profiles of local thicknesses of the $\mathrm{SiO}_{x} \mathrm{C}_{y} \mathrm{H}_{z}$ film. The solid lines correspond to result obtained by the ellipsometric method, the dashed lines correspond to results obtained by imagining spectroscopic reflectometry (ISR).
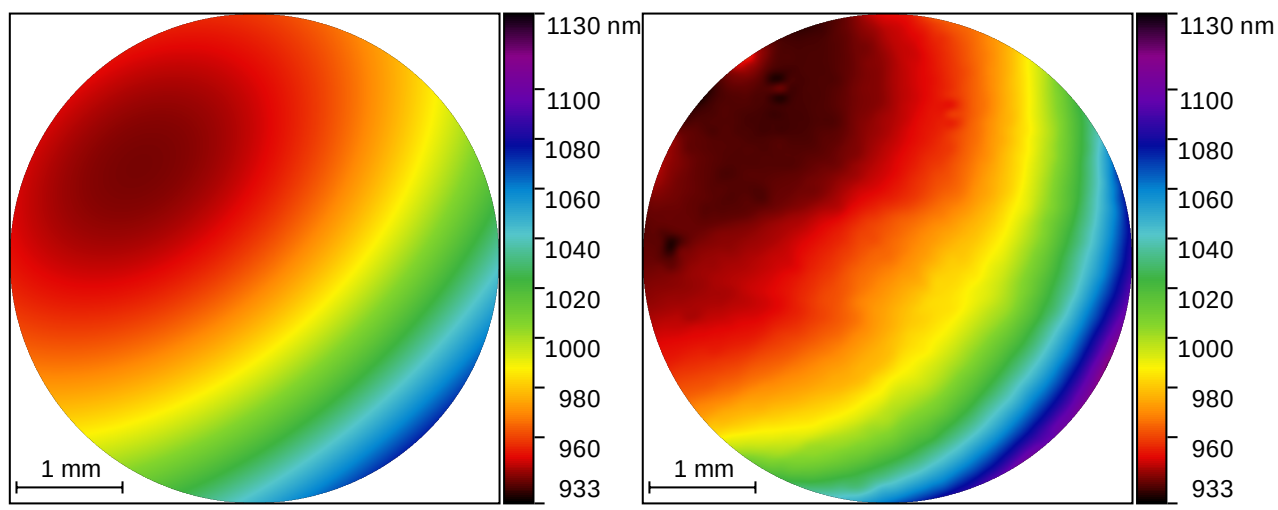

Figure 7. Maps of local thicknesses determined by the ellipsometric method (left) and by ISR (right) for the $\mathrm{SiO}_{x} \mathrm{C}_{y} \mathrm{H}_{z}$ film. 


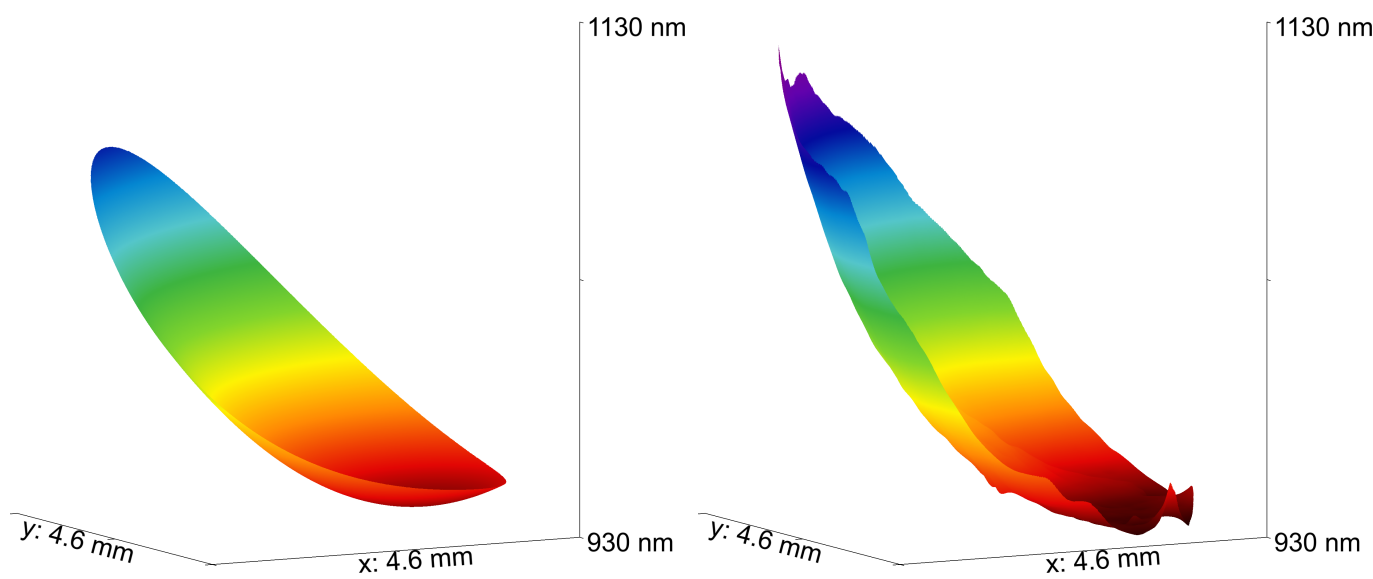

Figure 8. Three-dimensional representations of the shape of the upper boundary determined by the ellipsometric method (left) and by ISR (right) for the $\mathrm{SiO}_{x} \mathrm{C}_{y} \mathrm{H}_{z}$ film.
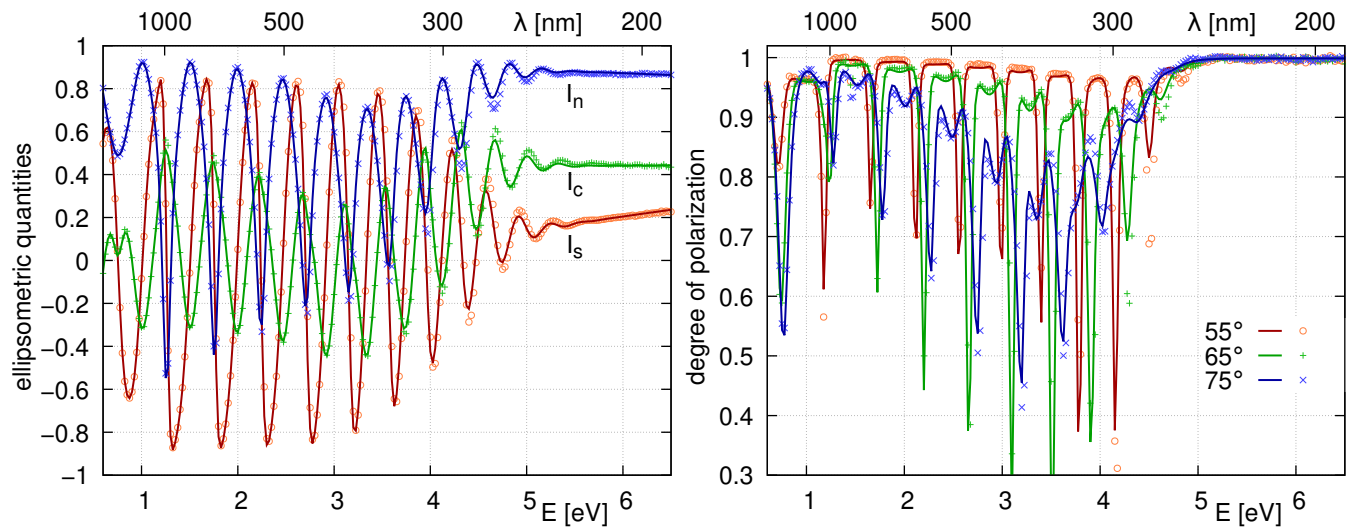

Figure 9. Agreement between the experimental and theoretical values for the associated ellipsometric parameters of the $\mathrm{SiO}_{x} \mathrm{C}_{y} \mathrm{H}_{z}$ film at incidence angle $70^{\circ}$ (left) and the degree of polarization at three selected angles of incidence (right). The plots correspond to azimuth angle $\beta=0^{\circ}$. The points represent the experimental values while the lines represent the theoretical values.

\subsection{Optical Characterization of the Inhomogeneous $\mathrm{SiN}_{x}$ Thin Films}

\subsubsection{Sample Preparation and Experimental Arrangements}

Three samples of thin films of $\mathrm{SiN}_{x}$ deposited onto double side polished silicon wafers were characterized. The deposition was carried out by the reactive magnetron sputtering of silicon target in argon-nitrogen atmosphere. During deposition the pressure was kept at constant value of $10^{-2} \mathrm{mbar}$ and the flow rates of argon and nitrogen were $80 \mathrm{sccm}$ and $5 \mathrm{sccm}$, respectively. The power supply for the magnetron was set at $100 \mathrm{~W}$. The films were deposited onto unheated silicon wafers. Three samples of the $\mathrm{SiN}_{x}$ thin films were deposited with different deposition times, i.e., for 30, 45 and $90 \mathrm{~min}$.

The associated ellipsometric parameters were again measured by the Horiba Jobin Yvon phase modulated ellipsometer (HORIBA Jobin Yvon, Irvine, CA, USA) for five incidence angles $55^{\circ}, 60^{\circ}, 65^{\circ}, 70^{\circ}$ and $75^{\circ}$ in the spectral region $0.6-6.3 \mathrm{eV}(197-2066 \mathrm{~nm})$.

Moreover, the spectral dependencies of reflectance at near-normal incidence were measured by a Perkin Elmer Lambda 1050 spectrophotometer (Perkin Elmer, Waltham, MA, USA) in the spectral range $0.7-6.5 \mathrm{eV}(190-1800 \mathrm{~nm})$. The ISR data were measured at normal incidence in the spectral range $1.7-4.5 \mathrm{eV}(275-700 \mathrm{~nm})$ with one pixel corresponding to $40 \times 40 \mu \mathrm{m}$.

\subsubsection{Influence of Boundary Roughness}

The influence of random roughness of the upper boundary on the optical quantities of the films is described by means of the SDT. This theory was used to derive the formulae for 
the optical quantities of homogeneous isotropic thin films with randomly rough boundaries (see e.g., [35-46]). The reflection coefficients of this inhomogeneous thin film are expressed by means of Formula (46). However, in this equation the reflection coefficients of the isotropic inhomogeneous thin film must be substituted by those for anisotropic inhomogeneous thin film $r_{I, q}$ expressed with Equation (55).

Moreover, the Fresnel coefficients of the upper boundary are given as follows:

$$
\begin{aligned}
& r_{\mathrm{U}, \mathrm{p}}=\frac{Y_{\mathrm{e}, 0}-Y_{\mathrm{p}, \mathrm{A}}}{Y_{\mathrm{e}, 0}+Y_{\mathrm{p}, \mathrm{A}}}, \quad t_{\mathrm{U}, \mathrm{p}}=\frac{\alpha_{\mathrm{A}}}{\alpha_{0}} \frac{2 Y_{\mathrm{p}, \mathrm{A}}}{Y_{\mathrm{e}, 0}+Y_{\mathrm{p}, \mathrm{A}}}, \quad r_{\mathrm{U}, \mathrm{p}}^{\prime}=\frac{Y_{\mathrm{p}, \mathrm{A}}-Y_{\mathrm{e}, 0}}{Y_{\mathrm{p}, \mathrm{A}}+Y_{\mathrm{e}, 0}}, \\
& t_{\mathrm{U}, \mathrm{p}}^{\prime}=\frac{\alpha_{0}}{\alpha_{\mathrm{A}}} \frac{2 Y_{\mathrm{e}, 0}}{Y_{\mathrm{p}, \mathrm{A}}+Y_{\mathrm{e}, 0}}, \quad r_{\mathrm{U}, \mathrm{s}}=\frac{Y_{\mathrm{o}, 0}-Y_{\mathrm{s}, \mathrm{A}}}{Y_{\mathrm{o}, 0}+Y_{\mathrm{s}, \mathrm{A}}}, \quad t_{\mathrm{U}, \mathrm{s}}=\frac{\alpha_{\mathrm{A}}}{\alpha_{0}} \frac{2 Y_{\mathrm{s}, \mathrm{A}}}{Y_{\mathrm{o}, 0}+Y_{\mathrm{s}, \mathrm{A}}}, \\
& r_{\mathrm{U}, \mathrm{s}}^{\prime}=\frac{Y_{\mathrm{s}, \mathrm{A}}-Y_{\mathrm{o}, 0}}{Y_{\mathrm{S}, \mathrm{A}}+Y_{\mathrm{o}, 0}}, \quad t_{\mathrm{U}, \mathrm{s}}^{\prime}=\frac{\alpha_{0}}{\alpha_{\mathrm{A}}} \frac{2 Y_{\mathrm{o}, 0}}{Y_{\mathrm{s}, \mathrm{A}}+Y_{\mathrm{e}, 0}},
\end{aligned}
$$

where admittances $Y_{\mathrm{e}, 0}, Y_{\mathrm{O}, 0}$ and quantity $\alpha_{0}$ at the upper boundary of the film are calculated by Equation (52) and the admittances $Y_{\mathrm{e}, \mathrm{A}}, Y_{\mathrm{O}, \mathrm{A}}$ and $\alpha_{\mathrm{A}}$ corresponding to the ambient are calculated using the following equations:

$$
\begin{aligned}
& k_{z, k}=k_{0} \sqrt{\varepsilon_{k}-n_{\mathrm{A}}^{2} \sin ^{2} \varphi_{\mathrm{A}}}=k_{0} n_{k} \cos \varphi_{k}, \quad \alpha_{k}=\frac{k_{z, k}}{k_{0} \sqrt{\varepsilon_{k}}}=\cos \varphi_{k}, \\
& Y_{\mathrm{s}, k}=\frac{k_{z, k}}{k_{0}}=n_{k} \cos \varphi_{k}, \quad Y_{\mathrm{p}, k}=\frac{k_{0} \varepsilon_{k}}{k_{z, k}}=\frac{n_{k}}{\cos \varphi_{k}}, \\
& \alpha_{\mathrm{A}}=\cos \varphi_{\mathrm{A}}, \\
& \Upsilon_{\mathrm{s}, \mathrm{A}}=n_{\mathrm{A}} \cos \varphi_{\mathrm{A}}, \quad Y_{\mathrm{p}, \mathrm{A}}=\frac{n_{\mathrm{A}}}{\cos \varphi_{\mathrm{A}}} .
\end{aligned}
$$

It should be noted that in case of the inhomogeneous thin film with the rough upper boundary it is necessary to omit matrix $B_{\mathrm{A}, 0}$ in calculating the elements of matrix $S^{\mathrm{I}}$. Moreover, the presented method assumes that the optical constants are constant within the range of random roughness of the upper boundaries.

\subsubsection{Dispersion Model}

The dielectric function of this $\mathrm{SiN}_{x}$ film is expressed by two contributions. Thus, this function is given as

$$
\varepsilon(E)=1+N_{\mathrm{vc}} \varepsilon_{\mathrm{dt}}^{0}(E)+N_{\mathrm{ut}} \varepsilon_{\mathrm{ut}}^{0}(E),
$$

where the first contribution represents the valence to conduction band transitions of electrons and the second contribution corresponds to the Urbach tail. This tail describes weak absorption below the band gap energy. Symbols $N_{\mathrm{vc}}$ and $N_{\mathrm{ut}}$ denote strengths of the band transitions and weak absorption corresponding to the Urbach tail. Functions $\varepsilon_{\mathrm{dt}}^{0}(E)$ and $\varepsilon_{\mathrm{ut}}^{0}(E)$ represent the normalized contributions to dielectric functions for the valence to conduction transitiosions and Urbach tail, respectively. The imaginary part of the normalized contribution to the dielectric function for the valence to conduction band transitions is expressed as follows [99]:

$$
\varepsilon_{\mathrm{i}, \mathrm{dt}}^{0}(E)=\frac{1}{1+A_{\mathrm{ex}}} \varepsilon_{\mathrm{i}, \mathrm{vc}}^{0}(E)+\frac{A_{\mathrm{ex}}}{1+A_{\mathrm{ex}}} \varepsilon_{\mathrm{i}, \mathrm{ex}}^{0}(E),
$$

where the first contribution is given as $[99,100]$

$$
\varepsilon_{\mathrm{i}, \mathrm{vc}}^{0}(E)=\frac{\left(E-E_{\mathrm{g}}\right)^{2}\left(E_{\mathrm{h}}-E\right)^{2}}{C_{\mathrm{v}} E^{2}} \Theta\left(E ; E_{\mathrm{g}}, E_{\mathrm{h}}\right)
$$


This term corresponds to the basic absorption band. The second term is expressed as follows [101,102]:

$$
\varepsilon_{\mathrm{i}, \mathrm{ex}}^{0}(E)=\frac{\left(E-E_{\mathrm{g}}\right)^{2}\left(E_{\mathrm{h}}-E\right)^{2}}{C_{\mathrm{v}} E^{2}\left[\left(E-E_{\mathrm{ex}}\right)^{2}+B_{\mathrm{ex}}^{2}\right]} \Theta\left(E ; E_{\mathrm{g}}, E_{\mathrm{h}}\right),
$$

where $A_{\mathrm{ex}}$ is used for to control the weights concerning $\varepsilon_{\mathrm{i}, \mathrm{vc}}^{0}$ and $\varepsilon_{\mathrm{i}, \mathrm{ex}}^{0}$.

The imaginary part of the normalized contribution representing the Urbach tail is expressed as:

$$
\begin{array}{r}
\varepsilon_{\mathrm{i}, \mathrm{ut}}^{0}(E)=\frac{1}{C_{\mathrm{ut}} E^{2}}\left[\left(1+\frac{\left(E-E_{\mathrm{g}}\right)\left(E_{\mathrm{m}}-E\right)}{E_{\mathrm{u}}\left(E_{\mathrm{m}}-E_{\mathrm{g}}\right)}\right) \Theta\left(E ; E_{\mathrm{g}}, E_{\mathrm{m}}\right)\right. \\
\left.+\mathrm{e}^{\frac{E-E_{\mathrm{g}}}{E_{\mathrm{u}}}} \Theta\left(E ; E_{\mathrm{g}} / 2, E_{\mathrm{g}}\right)+\mathrm{e}^{\frac{E_{\mathrm{m}}-E}{E_{\mathrm{u}}}} \Theta\left(E ; E_{\mathrm{m}}, E_{\mathrm{m}}+E_{\mathrm{g}} / 2\right)-\mathrm{e}^{-\frac{E_{\mathrm{g}}}{2 E_{\mathrm{u}}}}\right]
\end{array}
$$

where $E_{\mathrm{m}}=\left(E_{\mathrm{g}}+E_{\mathrm{h}}\right) / 2$ and $E_{\mathrm{u}}$ is the Urbach energy. Constants $C_{\mathrm{vc}}, C_{\mathrm{ex}}$ and $C_{\mathrm{ut}}$ must ensure the sum rule normalization condition (see e.g., $[99,101])$.

The dispersion model described above is utilized for the homogeneous isotropic thin films. The dispersion parameters corresponding to the thin films exhibiting uniaxial anisotropy must be separated to two sets, one set corresponds to the ordinary dielectric function and the latter set belongs to the extraordinary dielectric function. The sum rule (see e.g., [103]) must give the same value for the ordinary and extraordinary dielectric functions, which can be expressed using the following equation:

$$
N_{\mathrm{vc}, \mathrm{e}}+N_{\mathrm{ut}, \mathrm{e}}=N_{\mathrm{vc}, \mathrm{o}}+N_{\mathrm{ut}, \mathrm{o}}
$$

where indices e and o distinguish the strengths for the extraordinary and ordinary waves, respectively.

The inhomogeneity of the characterized $\mathrm{SiN}_{x}$ thin film is described by the profiles of the dispersion parameters $p_{\alpha, \gamma}$ expressed as

$$
p_{\alpha, \gamma}(z)=\mathrm{e}^{-z / \xi} p_{\alpha, \gamma}^{\mathrm{U}}+\left(1-\mathrm{e}^{-z / \xi}\right) p_{\alpha, \gamma}^{\infty}
$$

where index $\alpha$ distinguishes the individual parameters, $\xi$ is the parameter controlling the shape of the profile, $\gamma=\mathrm{e}, \mathrm{o}$ and $p_{\alpha, \gamma}^{\mathrm{U}}$ are the values of the parameters at the upper boundary of the inhomogeneous thin film. Symbol $p_{\alpha, \gamma}^{\infty}$ denotes the values of the parameters for $z \rightarrow \infty$.

\subsubsection{Data Processing}

The dispersion and structural models contain a large number of parameters that must be sought. If all of these parameters are considered to be independent then the problem of processing the experimental data is overparametrized. Therefore, it is necessary to reduce the number of these parameters. It is reasonable to expect that the inhomogeneity of the film and uniaxial anisotropy are relatively weak effects. Thus, one can assume that some dispersion parameters do not exhibit profile and some dispersion parameters have the identical value for the ordinary and extraordinary dielectric functions. Even when the number parameters are reduced in this way, there are still enough degrees of freedom left to successfully interpret the measured data. The concrete relations between the dispersion parameters are described in detail in [67]. The merit function that enables the performance of the efficient data processing by the LSM is also presented in [67]. Moreover, the modification of multisample method of the data processing was employed. This means that measured ellipsometric and reflectometric data were processed simultaneously for all three samples of the characterized nonstoichiometric $\mathrm{SiN}_{x}$ thin films (for details see [11,104-106]. By using this modification one can reduce correlations among the searched parameters which improves the fits of the experimental data. 


\subsubsection{Results and Discussion}

In Figure 10 the spectral dependencies of the optical constants of the inhomogeneous $\mathrm{SiN}_{x}$ films for the ordinary and extraordinary waves at the upper and lower boundaries are presented. The profiles of the optical constants of these films for the ordinary and extraordinary waves are depicted in Figure 11. In this figure it is apparent that the profiles of the inhomogeneous films corresponding to Samples 2 and 3 are similar while the profiles belonging to Sample 1 are somewhat different from the other two samples. This is also evident in Figure 10 on the spectral dependencies of the optical constants at the lower boundary that are nearly the same for Samples 2 and 3 while the refractive indices of Sample 1 are somewhat larger. However, note that the spectral dependencies of the optical constants at the upper boundary exhibit different behavior. The refractive indices corresponding to Samples 1 and 2 are almost identical while the refractive index of Sample 3 is a little smaller. Nevertheless, the difference between the spectral dependencies of the optical constants at the upper and lower boundaries are relatively small. This implies that the inhomogeneity in the optical constants of all three samples exhibits practically identical behavior. It should be emphasized that the optical constants change their values in the vicinity of the upper boundaries while the remaining parts of these inhomogeneous thin films are nearly homogeneous. From Figure 10 it is evident that refractive indices are smaller at the upper boundaries and higher at the lower boundaries. This reality implies that in the finishing part of the deposition of the characterized films technological conditions were considerably changed.

In the processing of the experimental data the influence of random roughness of the upper boundaries of the $\mathrm{SiN}_{x}$ thin films is described by the formula derived using the SDT. Within this derivation it was assumed that the optical constants are constant in the range of this roughness. This condition is not fulfilled for the samples of the $\mathrm{SiN}_{x}$ thin films characterized (see Figure 11). This means that the approach used to include the influence of roughness on the optical quantities should be considered to be only a rough approximation. However, roughness of the upper boundaries is slight (see Table 2) so that this approximation is permissible. This statement is also supported by the very good fits of the experimental data presented in Figure 12. Moreover, the rms values of sample 3 was determined by atomic force microscopy. This determined value is $7.5 \mathrm{~nm}$, which is in a relatively good agreement with the rms value found using the optical method (see Table 2). This result also supports a correctness of the results obtained in the optical characterization of the samples of the inhomogeneous $\mathrm{SiN}_{x}$ thin films.

In Table 2 the values of the structural parameters of the films are summarized. The small differences in values of thickness of the films determined using the separate processing of the ellipsometric and reflectometric data are seen. This could probably be caused by a very slight thickness nonuniformity or different systematic errors of both the optical techniques. Of course, both the effects can be combined. However, by using the ISR method it was found that samples were uniform in thickness within the accuracy of measurements. Thus, the thickness nonuniformity was not included in the structural model of the $\mathrm{SiN}_{x}$ films. In this table it is also seen that the rms values of the heights of roughness are very small for all three samples, i.e., these samples exhibit nanometric roughness of their upper boundaries. In the processing of the experimental data the optical constants of the silicon substrates were again fixed in values taken from [95].

The optical characterization of the inhomogeneous thin films of $\mathrm{SiN}_{x}$ can serve as an example of the successful optical characterization of thin films exhibiting a very complicated structure with enormous number of sought parameters. 

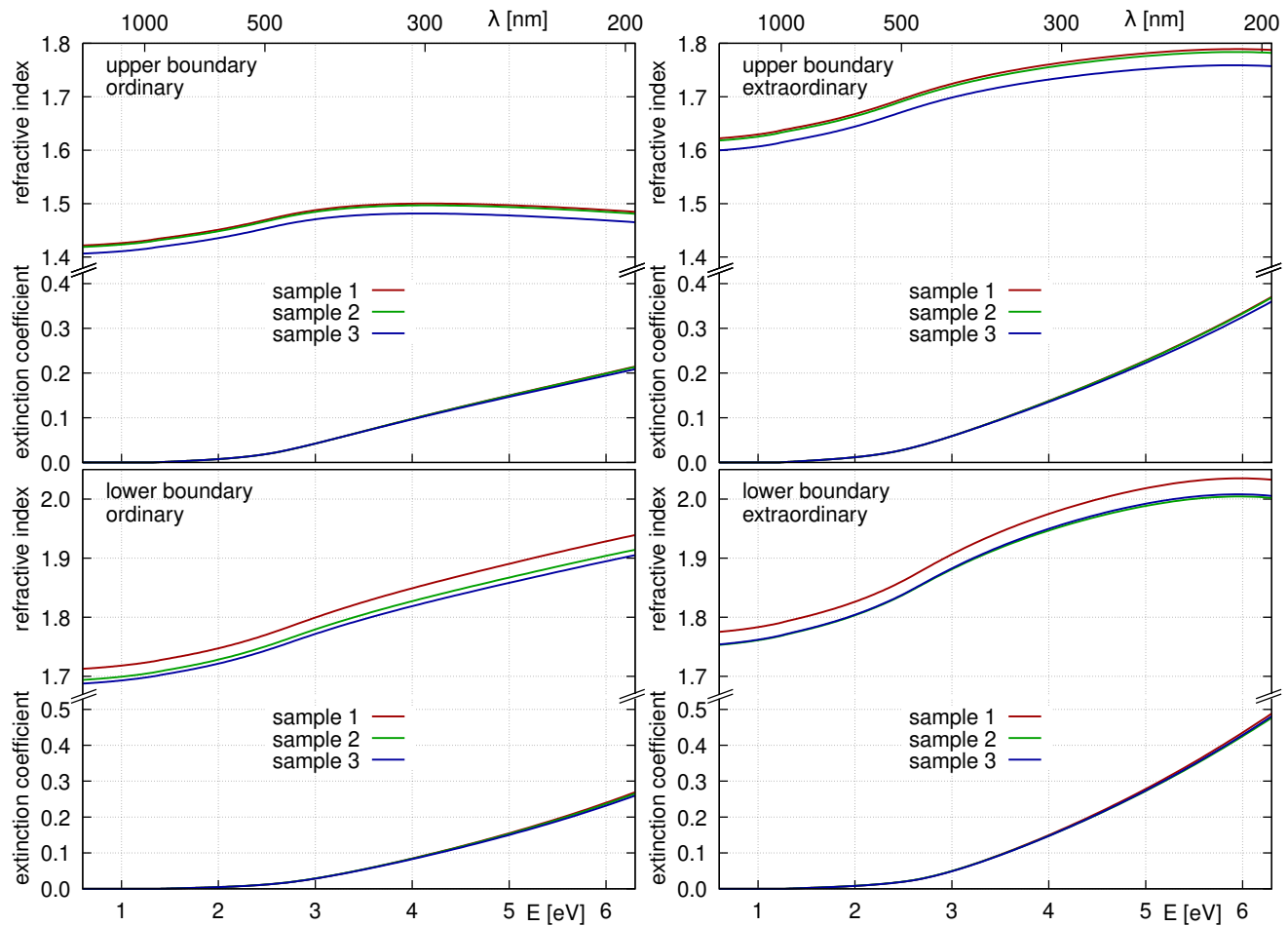

Figure 10. Spectral dependencies ofthe optical constants of the $\mathrm{SiN}_{x}$ film at the upper (top) and lower (bottom) boundaries for the ordinary (left) and extraordinary (right) waves.
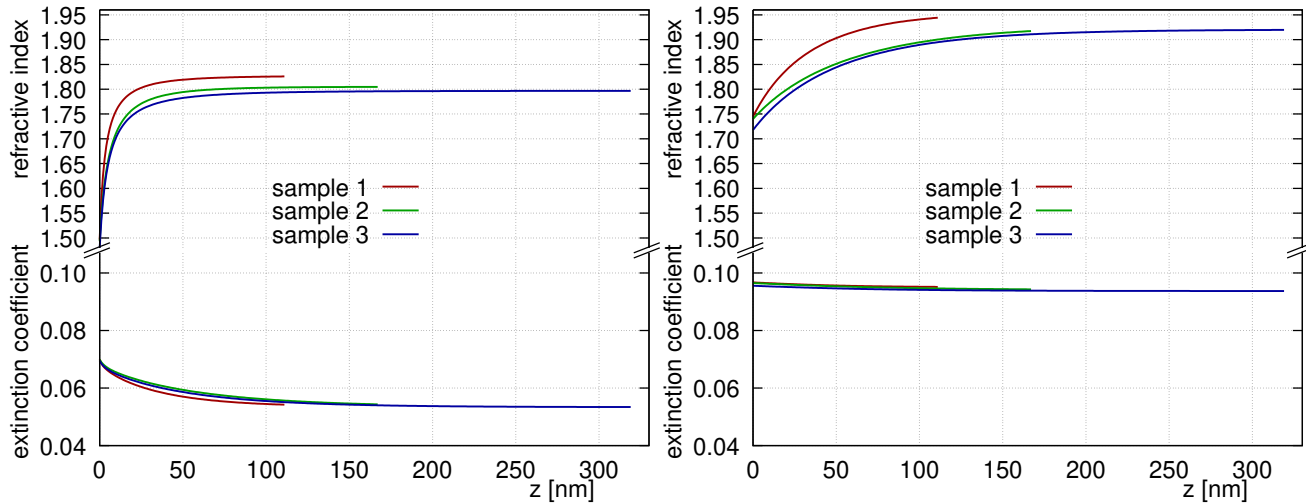

Figure 11. Profiles of the optical constants of the $\mathrm{SiN}_{x}$ film at $E=3.5 \mathrm{eV}$ for the ordinary (left) and extraordinary (right) waves.
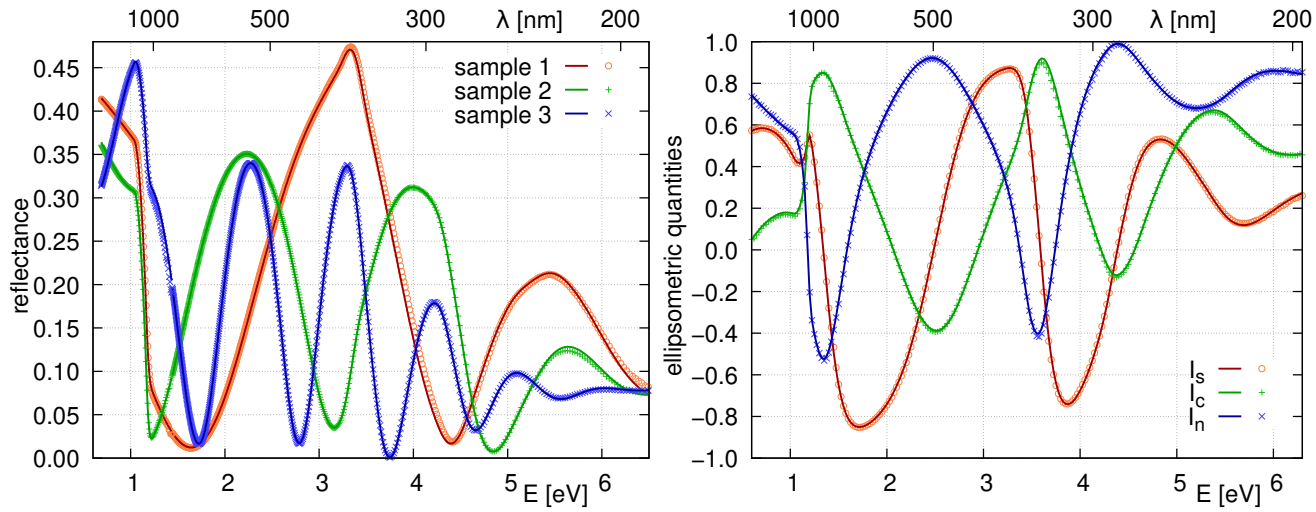

Figure 12. The spectral dependencies of reflectance for all three samples (left) and the ellipsometric quantities for sample 2 at incidence angle $70^{\circ}$ (right). The points represent the experimental values while the lines represent the theoretical values. 
Table 2. The values of the structural parameters.

\begin{tabular}{cccccc}
\hline & & & Sample 1 & Sample 2 & Sample 3 \\
\hline deposition time & $t$ & {$[\mathrm{~min}]$} & 30 & 45 & 90 \\
thickness ellipsometry & $d_{\mathrm{e}}$ & {$[\mathrm{nm}]$} & $111.21 \pm 0.05$ & $167.10 \pm 0.08$ & $323.9 \pm 0.1$ \\
thickness reflectance & $d_{\mathrm{r}}$ & {$[\mathrm{nm}]$} & $112.12 \pm 0.06$ & $167.91 \pm 0.07$ & $320.80 \pm 0.09$ \\
roughness (rms) & $\sigma$ & {$[\mathrm{nm}]$} & $3.0 \pm 0.2$ & $4.6 \pm 0.2$ & $6.3 \pm 0.2$ \\
profile parameter & $\xi$ & {$[\mathrm{nm}]$} & $39.3 \pm 1.8$ & $65.6 \pm 2.8$ & $57.6 \pm 2.6$ \\
\hline
\end{tabular}

\section{Conclusions}

This review paper is devoted to optics of the inhomogeneous thin films exhibiting defects that are often encountered in practice. The following defects are thus considered: transition layers, overlayers, thickness nonuniformity, boundary roughness and artificial (unwanted) uniaxial anisotropy with the optical axis perpendicular to the boundaries of the films. The approximations based on multilayer systems and multiple-beam interference model are used to describe the inhomogeneity of the thin films exhibiting these defects. In the theoretical part, the approaches utilized for including the individual defects into the formulae for the optical quantities of the inhomogeneous thin films are presented. The defects consisting in the transition layers and overlays are easily included into these formulae by means of the recursive or matrix formalisms. As for the thickness nonuniformity, the theoretical approach is more complicated. The inclusion of this defect into the mentioned formulae is performed using the averaging of the elements of the corresponding Mueller matrix. This averaging is carried out by means of a local thickness distribution density in the case of simple forms of this nonuniformity. The typical example of this simple nonuniformity is the wedge-shaped thickness nonuniformity. If the thickness nonuniformity is more complicated, this model is not satisfactory and a more complicated model describing the local thicknesses of the inhomogeneous thin film using a polynomial in coordinates along the surface of the film must be used. Random roughness of the boundaries of the inhomogeneous films also represents defect requiring relatively complicated mathematical approaches for its inclusion into the formulae of for the optical quantities. It is necessary to use approaches based on the scalar diffraction theory if randomly rough boundaries exhibit low spatial frequencies (locally smooth roughness) and perturbations theories such as the Rayleigh-Rice theory for roughness having moderate and high spatial frequencies. In this paper it is shown that for the film boundaries exhibiting a wide interval of spatial frequencies, it is possible to utilize the approximation based on the heuristic combination of both the theories. The defect consisting in the uniaxial anisotropy is included into formulae for reflection coefficients of the inhomogeneous thin films using the Yeh matrix formalism. The other matrix formalisms can also be utilized because in the case of the uniaxial anisotropy the extraordinary wave corresponds to the p-polarization and ordinary wave corresponds to the s-polarization.

The paper also contains two selected examples of the optical characterization of the inhomogeneous thin films exhibiting the combinations of the defects mentioned above. The optical characterization of one inhomogeneous polymer-like thin film $\left(\mathrm{SiO}_{x} \mathrm{C}_{y} \mathrm{H}_{z}\right)$ prepared by the PECVD onto the silicon single crystal substrates exhibiting the combination of the transition layer and thickness nonuniformity is performed. This film exhibits the complicated thickness nonuniformity differing from the wedge-shaped model. Therefore, thickness nonuniformity had to be included into the formulae for the ellipsometric parameters using the approach with the local thicknesses given by the polynomial in the coordinates along the film surface. The complete optical characterization of the polymerlike thin film consisting in determining all the parameters of this film is carried out by means of variable angle spectroscopic ellipsometry. This means that the spectral dependencies of the optical constants at upper and lower boundaries, profiles of these constants and values of the coefficients of the quadratic polynomial describing the geometry of the surface of the upper boundary were determined. The results concerning thickness nonuniformity were confirmed by ISR. The complete optical characterization of the three samples 
of the inhomogeneous thin films of nonstoichiometric silicon nitride $\left(\mathrm{SiN}_{x}\right)$ prepared by magnetron sputtering onto the same substrates is performed by the combined method of variable angle spectroscopic ellipsometry and spectroscopic reflectomery applied at the near-normal incidence of light. These samples exhibit the combination of random roughness of the upper boundaries and uniaxial anisotropy. Boundary roughness was described using the SDT. Inhomogeneity in the optical constants was expressed by the approximate approach based on the multilayer system. Uniaxial anisotropy was described by the Yeh formalism. In the processing of the experimental data the multiple-sample method is used to determine all the parameters of the films, i.e., spectral dependencies of the optical constants at the upper and lower boundaries for the ordinary and extraordinary waves, profiles of the optical constants for both the waves and mean thickness values of these films were determined. Moreover, the rms values of the heights of boundary roughness were determined. In the multiple-sample method, the experimental data of the three samples were processed simultaneously, which enables us to increase the efficiency of the optical characterization of the inhomogeneous $\mathrm{SiN}_{x}$ thin films. The ISR method was used to verify the results of the optical characterization achieved for the inhomogeneous nonstoichiometric silicon nitride thin films.

The examples of the optical characterization of the inhomogeneous thin films of $\mathrm{SiO}_{x} \mathrm{C}_{y} \mathrm{H}_{z}$ and $\mathrm{SiN}_{x}$ also show that the processing of the experimental data is very difficult because the formulae for the inhomogeneous films including thickness nonuniformity and boundary roughness are complicated from the mathematical point of view. The integrals occurring in these formulae must be solved using the sophisticated numerical methods based on the Chebyshev approximation and Gaussian quadrature. The processing of the experimental data without using these sophisticated numerical methods is impossible from the practical point of view. However, the inhomogeneous thin films with defects will be encountered more and more frequently in practice which is caused by a progress in various branches of the fundamental and applied researches such as in the semiconductor industry, optics industry, solar energetics, etc. Therefore, one can expect that in the future the methods described above will be utilized more frequently in spite of their difficulties.

The main aim of this review paper is to summarize the theoretical approaches enabling one to derive the formulae for the optical quantities of the inhomogeneous thin films exhibiting the defects often occurring in practice, together with illustrating the examples of the optical characterization of the inhomogeneous thin films with the selected defects. The other reason why we decided to write this review paper is our desire to inspire the researchers to utilize the presented methods within the optical characterization of inhomogeneous thin films exhibiting defects.

Author Contributions: Conceptualization, I.O. methodology, J.V., I.O.; software, J.V.; investigation, J.V., M.Č. All authors have read and agreed to the published version of the manuscript.

Funding: This work was supported by the project LM2018097 funded by Ministry of Education, Youth and Sports of the Czech Republic.

Institutional Review Board Statement: Not applicable.

Informed Consent Statement: Not applicable.

Data Availability Statement: The data presented in this study are available on request from the corresponding author.

Conflicts of Interest: The authors declare no conflict of interest. The funders had no role in the design of the study; in the collection, analyses, or interpretation of data; in the writing of the manuscript, or in the decision to publish the results. 


\section{References}

1. Lohner, T.; Kumar, K.J.; Petrik, P.; Subrahmanyam, A.; Bársony, I. Optical analysis of room temperature magnetron sputtered ITO films by reflectometry and spectroscopic ellipsometry. J. Mater. Res. 2014, 29, 1528-1536. [CrossRef]

2. Vašiček, A. Polarimetric Methods for the Determination of the Refractive Index and the Thickness of Thin Films on Glass. J. Opt. Soc. Am. 1947, 37, 145-153. [CrossRef] [PubMed]

3. Heavens, O.S.; Liddell, H.M. Influence of Absorption on Measurement of Refractive Index of Films. Appl. Opt. 1965, 4, 629-630. [CrossRef]

4. Vedam, K.; Knausenberger, W.; Lukes, F. Ellipsometric Method for the Determination of All the Optical Parameters of the System of an Isotropic Nonabsorbing Film on an Isotropic Absorbing Substrate. Optical Constants of Silicon. J. Opt. Soc. Am. 1969, 59, 64-71. [CrossRef]

5. Ritter, E. Optical film materials and their applications. Appl. Opt. 1976, 15, 2318-2327. [CrossRef] [PubMed]

6. Pulker, H.K.; Paesold, G.; Ritter, E. Refractive indices of $\mathrm{TiO}_{2}$ films produced by reactive evaporation of various titanium-oxygen phases. Appl. Opt. 1976, 15, 2986-2991. [CrossRef] [PubMed]

7. Bennett, J.M.; Pelletier, E.; Albrand, G.; Borgogno, J.P.; Lazarides, B.; Carniglia, C.K.; Schmell, R.A.; Allen, T.H.; Tuttle-Hart, T.; Guenther, K.H.; et al. Comparison of the properties of titanium dioxide films prepared by various techniques. Appl. Opt. 1989, 28, 3303-3317. [CrossRef]

8. Ohlídal, I.; Navrátil, K.; Schmidt, E. Simple method for the complete optical analysis of very thick and weakly absorbing films: Application to magnetic garnet-films. Appl. Phys. A 1982, 29, 157-162. [CrossRef]

9. Franta, D.; Ohlídal, I.; Petrýdes, D. Optical characterization of $\mathrm{TiO}_{2}$ thin films by the combined method of spectroscopic ellipsometry and spectroscopic photometry. Vacuum 2005, 80, 159-162. [CrossRef]

10. Franta, D.; Ohlídal, I.; Buršíková, V.; Zajíčková, L. Optical properties of diamond-like carbon films containing $\mathrm{SiO}_{x}$ studied by the combined method of spectroscopic ellipsometry and spectroscopic reflectometry. Thin Solid Films 2004, 455-456, 393-398. [CrossRef]

11. Ohlídal, I.; Franta, D.; Pinčík, E.; Ohlídal, M. Complete Optical Characterization of the $\mathrm{SiO}_{2} / \mathrm{Si}$ System by Spectroscopic Ellipsometry Spectroscopic Reflectometry and Atomic Force Microscopy. Surf. Interface Anal. 1999, 28, 240-244. [CrossRef]

12. Franta, D.; Ohlídal, I.; Frumar, M.; Jedelský, J. Optical Characterization of Chalcogenide Thin Films. Appl. Surf. Sci. 2001, 175-176, 555-561. [CrossRef]

13. Borgogno, J.P.; Pelletier, E. Determination of the extinction coefficient of dielectric thin films from spectrophotometric measurements. Appl. Opt. 1989, 28, 2895-2901. [CrossRef] [PubMed]

14. Lukeš, F.; Knausenberger, W.; Vedam, K. Ellipsometric liquid immersion method for the determination of all the optical parameters of the system: Nonabsorbing film on an absorbing substrate. Surf. Sci. 1969, 16, 112-125. [CrossRef]

15. Lukěs, F.; Schmidt, E. Another method for the determination of silicon oxide thickness. Solid-State Electron. 1967, 10, 264-266. [CrossRef]

16. Hacskaylo, M. Determination of the Refractive Index of Thin Dielectric Films. J. Opt. Soc. Am. 1964, 54, 198-203. [CrossRef]

17. Manifacier, J.C.; Gasiot, J.; Fillard, J.P. A simple method for the determination of the optical constants n, k and the thickness of a weakly absorbing thin film. J. Phys. E Sci. Instrum. 1976, 9, 1002-1004. [CrossRef]

18. Lukeš, F. Oxidation of Si and GaAs in air at room temperature. Surf. Sci. 1972, 30, 91-100. [CrossRef]

19. Stenzel, O.; Wilbrandt, S.; Kaiser, N.; Vinnichenko, M.; Munnik, F.; Kolitsch, A.; Chuvilin, A.; Kaiser, U.; Ebert, J.; Jakobs, S.; et al. The correlation between mechanical stress, thermal shift and refractive index in $\mathrm{HfO}_{2}, \mathrm{Nb}_{2} \mathrm{O}_{5}, \mathrm{Ta}_{2} \mathrm{O}_{5}$ and $\mathrm{SiO}_{2}$ layers and its relation to the layer porosity. Thin Solid Films 2009, 517, 6058-6068. [CrossRef]

20. Jacobsson, R. Matching a Multilayer Stack to a High-Refractive Index Substrate by Means of an Inhomogeneous Layer. J. Opt. Soc. Am. 1964, 54, 422-423. [CrossRef]

21. Charmet, J.C.; de Gennes, P.G. Ellipsometric formulas for an inhomogeneous layer with arbitrary refractive-index profile. J. Opt. Soc. Am. 1983, 73, 1777-1784. [CrossRef]

22. Carniglia, C.K. Ellipsometric calculations for nonabsorbing thin films with linear refractive-index gradients. J. Opt. Soc. Am. A 1990, 7, 848-856. [CrossRef]

23. Sheldon, B.; Haggerty, J.S.; Emslie, A.G. Exact computation of the reflectance of a surface layer of arbitrary refractive-index profile and an approximate solution of the inverse problem. J. Opt. Soc. Am. 1982, 72, 1049-1055. [CrossRef]

24. Monaco, S.F. Reflectance of an Inhomogeneous Thin Film. J. Opt. Soc. Am. 1961, 51, 280-282. [CrossRef]

25. Adamson, P. Reflection of light in a long-wavelength approximation from an N-layer system of inhomogeneous dielectric films and optical diagnostics of ultrathin layers. II. Transparent substrate. J. Opt. Soc. Am. B 2004, 21, 645-654. [CrossRef]

26. Debieu, O.; Nalini, R.P.; Cardin, J.; Portier, X.; Perrière, J.; Gourbilleau, F. Structural and optical characterization of pure Si-rich nitride thin films. Nanoscale Res. Lett. 2013, 8, 31. [CrossRef] [PubMed]

27. Bovard, B.G. Rugate filter design: The modified Fourier transform technique. Appl. Opt. 1990, 29, 24-30. [CrossRef]

28. Boivin, G.; St.-Germain, D. Synthesis of gradient-index profiles corresponding to spectral reflectance derived by inverse Fourier transform. Appl. Opt. 1987, 26, 4209-4213. [CrossRef]

29. Franta, D.; Ohlídal, I.; Klapetek, P.; Pokorný, P.; Ohlídal, M. Analysis of Inhomogeneous Thin Films of $\mathrm{ZrO}_{2}$ by the Combined Optical Method and Atomic Force Microscopy. Surf. Interface Anal. 2001, 32, 91-94. [CrossRef] 
30. Nečas, D.; Franta, D.; Ohlídal, I.; Poruba, A.; Wostrý, P. Ellipsometric characterization of inhomogeneous non-stoichiometric silicon nitride films. Surf. Interface Anal. 2013, 45, 1188-1192. [CrossRef]

31. Kildemo, M. Real-Time Monitoring and Growth Control of Si-Gradient-Index Structures by Multiwavelength Ellipsometry. Appl. Opt. 1998, 37, 113-124. [CrossRef] [PubMed]

32. Kildemo, M.; Brenot, R.; Drévillon, B. Spectroellipsometric method for process monitoring semiconductor thin films and interfaces Appl. Opt. 1998, 37, 5145-5149. [CrossRef] [PubMed]

33. Vohánka, J.; Ohlídal, I.; Ženíšek, J.; Vašina, P.; Čermák, M.; Franta, D. Use of the Richardson extrapolation in optics of inhomogeneous layers: Application to optical characterization. Surf. Interface Anal. 2018, 50, 757-765. [CrossRef]

34. Li, D.; Goullet, A.; Carette, M.; Granier, A.; Landesman, J.P. Effect of growth interruptions on $\mathrm{TiO}_{2}$ films deposited by plasma enhanced chemical vapour deposition. Mater. Chem. Phys. 2016, 182, 409-417. [CrossRef]

35. Ohlídal, I.; Lukeš, F. Ellipsometric Parameters of Rough Surfaces and of a System Substrate-Thin Film with Rough Boundaries. Opt. Acta 1972, 19, 817-843. [CrossRef]

36. Ohlídal, I.; Navrátil, K.; Lukeš, F. Reflection of Light by a System of Nonabsorbing Isotropic Film-Nonabsorbing Isotropic Substrate with Randomly Rough Boundaries. J. Opt. Soc. Am. 1971, 61, 1630-1639. [CrossRef]

37. Morris, I.L.; Jenkins, T.E. The effect of long-correlation-length surface roughness on the ellipsometric parameters of reflected light. Europhys. Lett. 1996, 34, 55-61. [CrossRef]

38. Carniglia, C.K. Scalar scattering theory for multilayer optical coatings. Opt. Eng. 1979, 18, 104-115. [CrossRef]

39. Zavislan, J.M. Angular scattering from optical interference coatings: Scalar scattering predictions and measurements. Appl. Opt. 1991, 30, 2224-2244. [CrossRef]

40. Bauer, J. Optical properties, band gap, and surface roughness of $\mathrm{Si}_{3} \mathrm{~N}_{4}$. Phys. Status Solidi A 1977, 39, 411-418. [CrossRef]

41. Bauer, J.; Biste, L.; Bolze, D. Optical properties of aluminium nitride prepared by chemical and plasmachemical vapour deposition. Phys. Status Solidi A 1977, 39, 173-181. [CrossRef]

42. Nagata, K.; Nishiwaki, J. Reflection of Light from Filmed Rough Surface: Determination of Film Thickness and rms Roughness. Jpn. J. Appl. Phys. 1967, 6, 251-257. [CrossRef]

43. Ohlídal, I.; Vižd'a, F.; Ohlídal, M. Optical analysis by means of spectroscopic reflectometry of single and double layers with correlated randomly rough boundaries. Opt. Eng. 1995, 34, 1761-1768.

44. Ohlídal, I.; Lukeš, F.; Navrátil, K. Rough silicon surfaces studied by optical methods. Surf. Sci. 1974, 45, 91-116. [CrossRef]

45. Eastman, J.M. Scattering by all-dielectric multilayer bandpass filters and mirrors for lasers. In Physics of Thin Films; Hass, G., Francombe, M.H., Eds.; Academic Press: New York, NY, USA, 1978; Volume 10, pp. 167-226.

46. Ohlídal, I.; Franta, D.; Nečas, D. Improved combination of scalar diffraction theory and Rayleigh-Rice theory and its application to spectroscopic ellipsometry of randomly rough surfaces. Thin Solid Films 2014, 571, 695-700. [CrossRef]

47. Amra, C.; Apfel, J.H.; Pelletier, E. Role of interface correlation in light scattering by a multiplayer. Appl. Opt. 1992, $31,3134-3151$. [CrossRef]

48. Ohlídal, I.; Franta, D. Matrix Formalism for Imperfect Thin Films. Acta Phys. Slov. 2000, 50, 489-500.

49. Pisarkiewicz, T. Reflection spectrum for a thin film with non-uniform thickness. J. Phys. D Appl. Phys. 1994, 27, 160-164. [CrossRef]

50. Pisarkiewicz, T.; Stapinski, T.; Czternastek, H.; Rava, P. Inhomogeneity of amorphous silicon thin films from optical transmission and reflection measurements. J. Non-Cryst. Solids 1991, 137, 619-622. [CrossRef]

51. Nečas, D.; Ohlídal, I.; Franta, D. The reflectance of non-uniform thin films. J. Opt. A Pure Appl. Opt. 2009, 11, 045202. [CrossRef]

52. Nečas, D.; Ohlídal, I.; Franta, D. Variable-angle spectroscopic ellipsometry of considerably non-uniform thin films. J. Opt. 2011, 13, 085705. [CrossRef]

53. Ohlídal, I.; Ohlídal, M.; Nečas, D.; Franta, D.; Buršíková, V. Optical characterisation of $\mathrm{SiO}_{x} \mathrm{C}_{y} \mathrm{H}_{z}$ thin films non-uniform in thickness using spectroscopic ellipsometry, spectroscopic reflectometry and spectroscopic imaging reflectometry. Thin Solid Films 2011, 519, 2874-2876. [CrossRef]

54. Vohánka, J.; Franta, D.; Čermák, M.; Homola, V.; Buršíková, V.; Ohlídal, I. Ellipsometric characterization of highly non-uniform thin films with the shape of thickness non-uniformity modeled by polynomials. Opt. Express 2020, 28, 5492-5506. [CrossRef] [PubMed]

55. Vohánka, J.; Šustek, Š.; Buršíková, V.; Šklíbová, V.; Šulc, V.; Homola, V.; Franta, D.; Čermák, M.; Ohlídal, M.; Ohlídal, I. Determining shape of thickness non-uniformity using variable-angle spectroscopic ellipsometry. Appl. Surf. Sci. 2020, 534, 147625. [CrossRef]

56. Pittal, S.; Snyder, P.G.; Ianno, N.J. Ellipsometry study of non-uniform lateral growth of ZnO thin films. Thin Solid Films 1993, 233, 286-288. [CrossRef]

57. Richter, U. Application of the degree of polarization to film thickness gradients. Thin Solid Films 1998, 313-314, 102-107. [CrossRef]

58. Ohlídal, M.; Ohlídal, I.; Klapetek, P.; Nečas, D.; Majumdar, A. Measurement of the thickness distribution and optical constants of non-uniform thin films. Meas. Sci. Technol. 2011, 22, 085104. [CrossRef]

59. Ohlídal, M.; Ohlídal, I.; Franta, D.; Králík, T.; Jákl, M.; Eliáš, M. Optical characterization of thin films non-uniform in thickness by a multiple-wavelength reflectance method. Surf. Interface Anal. 2002, 34, 660-663. [CrossRef] 
60. Nečas, D.; Čudek, V.; Vodák, J.; Ohlídal, M.; Klapetek, P.; Benedikt, J.; Rügner, K.; Zajíčková, L. Mapping of properties of thin plasma jet films using imaging spectroscopic reflectometry. Meas. Sci. Technol. 2014, 25, 115201. [CrossRef]

61. Ohlídal, M.; Vodák, J.; Nečas, D. Optical Characterization of Thin Films by Means of Imaging Spectroscopic Reflectometry. In Optical Characterization of Thin Solid Films; Stenzel, O., Ohlídal, M., Eds.; Springer International Publishing: Cham, Switzerland, 2018; pp. 107-141.

62. Taft, E.; Cordes, L. Optical Evidence for a Silicon-Silicon Oxide Interlayer. J. Electrochem. Soc. 1979, 126, 131-134. [CrossRef]

63. Aspnes, D.E.; Theeten, J.B. Dielectric function of $\mathrm{Si}_{-} \mathrm{SiO}_{2}$ and $\mathrm{Si}_{-} \mathrm{Si}_{3} \mathrm{~N}_{4}$ mixtures. J. Appl. Phys. 1979, 50, 4928-4935. [CrossRef]

64. Ohlídal, M.; Ohlídal, I.; Lukeš, F. Ellipsometric studies of polished silicon surfaces. Surf. Sci. 1976, 55, 467-476. [CrossRef]

65. Ohlídal, I.; Franta, D.; Nečas, D. Ellipsometric and reflectometric characterization of thin films exhibiting thickness non-uniformity and boundary roughness. Appl. Surf. Sci. 2017, 421, 687-696. [CrossRef]

66. Ohlídal, I.; Vohánka, J.; Buršíková, V.; Ženíšek, J.; Vašina, P.; Čermák, M.; Franta, D. Optical characterization of inhomogeneous thin films containing transition layers using the combined method of spectroscopic ellipsometry and spectroscopic reflectometry based on multiple-beam interference model. J. Vac. Sci. Technol. B 2019, 37, 062921. [CrossRef]

67. Vohánka, J.; Ohlídal, I.; Ohlídal, M.; Šustek, V.; Čermák, M.; Šulc, V.; Vašina, P.; Ženíšek, J.; Franta, D. Optical Characterization of Non-Stoichiometric Silicon Nitride Films Exhibiting Combined Defects. Coatings 2019, 9, 416. [CrossRef]

68. Ohlídal, I.; Vohánka, J.; Buršíková, V.; Franta, D.; Čermák, M. Spectroscopic ellipsometry of inhomogeneous thin films exhibiting thickness non-uniformity and transition layers. Opt. Express 2020, 28, 160-174. [CrossRef]

69. Jacobsson, R. Inhomogeneous and Coevaporated Homogeneous Films for Optical Applications. In Physics of Thin Films; Hass, G., Francombe, M.H., Hoffman, R.W., Eds.; Academic Press: New York, NY, USA, 1975; Volume 8, pp. 51-98.

70. Ohlídal, I.; Čermák, M.; Vohánka, J. Optical Characterization of Thin Films Exhibiting Defects. In Optical Characterization of Thin Solid Films; Stenzel, O., Ohlídal, M., Eds.; Springer International Publishing: Cham, Switzerland, 2018; pp. $271-313$.

71. Lekner, J. Exact reflection amplitudes for the Rayleigh profile. Phys. A 1982, 116, 235-247. [CrossRef]

72. Vašíček, A. Optics of Thin Films; North-Holland: Amsterdam, The Netherlands, 1960.

73. Knittl, Z. Optics of Thin Films; Wiley: London, UK, 1976.

74. Stenzel, O. The Physics of Thin Film Optical Spectra; Surface Sciences; Springer: Berlin, Germany, 2005.

75. Kildemo, M.; Hunderi, O.; Drévillon, B. Approximation of reflection coefficients for rapid real-time calculation of inhomogeneous films. J. Opt. Soc. Am. A 1997, 14, 931-939. [CrossRef]

76. Ohlídal, I.; Vohánka, J.; Mistrík, J.; Čermák, M.; Vižd'a, F.; Franta, D. Approximations of reflection and transmission coefficients of inhomogeneous thin films based on multiple-beam interference model. Thin Solid Films 2019, 692, 137189. [CrossRef]

77. Ohlídal, I.; Vohánka, J.; Čermák, M.; Franta, D. Ellipsometry of Layered Systems. In Optical Characterization of Thin Solid Films; Stenzel, O., Ohlídal, M., Eds.; Springer International Publishing: Cham, Switzerland, 2018; pp. 233-267.

78. Azzam, R.M.A. Mueller-matrix ellipsometry: A review. In Polarization: Measurement, Analysis, and Remote Sensing, Proceedings of the SPIE, San Diego, CA, USA, 27 July 1997; SPIE: Bellingham, WA, USA, 1997; Volume 3121, pp. $396-405$.

79. Garcia-Caurel, E.; Ossikovski, R.; Foldyna, M.; Pierangelo, A.; Drévillon, B.; De Martino, A. Advanced Mueller Ellipsometry Instrumentation and Data Analysis. In Ellipsometry at the Nanoscale; Losurdo, M., Hingerl, K., Eds.; Springer: Berlin/Heidelberg, Germany, 2013; Chapter 2, pp. 31-143.

80. Goldstein, D. Polarized Light, Revised and Expanded; Dekker: New York, NY, USA, 2003.

81. Forcht, K.; Gombert, A.; Joerger, R.; Köhl, M. Incoherent superposition in ellipsometric measurements. Thin Solid Films 1997, 302, 43-50. [CrossRef]

82. Ossikovski, R.; Kildemo, M.; Stchakovsky, M.; Mooney, M. Anisotropic Incoherent Reflection Model for Spectroscopic Ellipsometry of a Thick Semitransparent Anisotropic Substrate. Appl. Opt. 2000, 39, 2071-2077. [CrossRef] [PubMed]

83. Stroud, A.H.; Secrest, D. Gaussian Quadrature Formulas; Prentice-Hall Series in Automatic Computation; Prentice-Hall: Englewood Cliffs, NJ, USA, 1966.

84. Ohlídal, I.; Franta, D. Ellipsometry of Thin Film Systems. In Progress in Optics; Wolf, E., Ed.; Elsevier: Amsterdam, The Netherlands, 2000; Volume 41, pp. 181-282.

85. Rayleigh, J.W.S.B. The Theory of Sound; Macmillan and Company: London, UK, 1878.

86. Rice, S.O. Reflection of Electromagnetic Waves from Slightly Rough Surfaces. Commun. Pure Appl. Math. 1951, 4, 351-378. [CrossRef]

87. Franta, D.; Ohlídal, I. Ellipsometric parameters and reflectances of thin films with slightly rough boundaries. J. Mod. Opt. 1998, 45, 903-934. [CrossRef]

88. Čermák, M.; Vohánka, J.; Ohlídal, I.; Franta, D. Optical quantities of multi-layer systems with randomly rough boundaries calculated using the exact approach of the Rayleigh-Rice theory. J. Mod. Opt. 2018, 65, 1720-1736. [CrossRef]

89. Vohánka, J.; Čermák, M.; Franta, D.; Ohlídal, I. Efficient method to calculate the optical quantities of multi-layer systems with randomly rough boundaries using the Rayleigh-Rice theory. Phys. Scr. 2019, 94, 045502. [CrossRef]

90. Franta, D.; Ohlídal, I.; Nečas, D. Influence of cross-correlation effects on the optical quantities of rough films. Opt. Express 2008, 16, 7789-7803. [CrossRef]

91. Ohlídal, I.; Vohánka, J.; Čermák, M.; Franta, D. Combination of spectroscopic ellipsometry and spectroscopic reflectometry with including light scattering in the optical characterization of randomly rough silicon surfaces covered by native oxide layers. Surf. Topogr. Metrol. Prop. 2019, 7, 045004. [CrossRef] 
92. Nečas, D.; Ohlídal, I.; Franta, D.; Ohlídal, M.; Vodák, J. Simultaneous determination of optical constants, local thickness and roughness of ZnSe thin films by imaging spectroscopic reflectometry. J. Opt. 2016, 18, 015401. [CrossRef]

93. Nečas, D.; Ohlídal, I. Consolidated series for efficient calculation of the reflection and transmission in rough multilayers. Opt. Express 2014, 22, 4499-4515. [CrossRef]

94. Yeh, P. Optics of anisotropic layered media: A new $4 \times 4$ matrix algebra. Surf. Sci. 1980, 96, 41-53. [CrossRef]

95. Franta, D.; Dubroka, A.; Wang, C.; Giglia, A.; Vohánka, J.; Franta, P.; Ohlídal, I. Temperature-dependent dispersion model of float zone crystalline silicon. Appl. Surf. Sci. 2017, 421, 405-419. [CrossRef]

96. Pierce, D.T.; Spicer, W.E. Electronic Structure of Amorphous Si from Photoemission and Optical Studies. Phys. Rev. B 1972, 5, 3017-3029. [CrossRef]

97. Franta, D.; Nečas, D.; Ohlídal, I.; Giglia, A. Dispersion model for optical thin films applicable in wide spectral range. Optical Systems Design 2015: Optical Fabrication, Testing, and Metrology V. Proc. SPIE 2015, 9628, 96281U.

98. Campi, D.; Coriasso, C. Prediction of Optical Properties of Amorphous Tetrahedrally Bounded Materials. J. Appl. Phys. 1988, 64, 4128-4134. [CrossRef]

99. Franta, D.; Vohánka, J.; Čermák, M. Universal Dispersion Model for Characterisation of Thin Films Over Wide Spectral Range. In Optical Characterization of Thin Solid Films; Stenzel, O., Ohlídal, M., Eds.; Springer International Publishing: Cham, Switzerland, 2018; pp. 31-82.

100. Franta, D.; Nečas, D.; Ohlídal, I. Universal dispersion model for characterization of optical thin films over wide spectral range: Application to hafnia. Appl. Opt. 2015, 54, 9108-9119. [CrossRef]

101. Franta, D.; Nečas, D.; Zajíčková, L.; Ohlídal, I.; Stuchlík, J.; Chvostová, D. Application of sum rule to the dispersion model of hydrogenated amorphous silicon. Thin Solid Films 2013, 539, 233-244. [CrossRef]

102. Franta, D.; Zajíčková, L.; Ohlídal, I.; Janča, J.; Veltruská, K. Optical characterization of diamond like carbon films using multi-sample modification of variable angle spectroscopic ellipsometry. Diam. Relat. Mat. 2002, 11, 105-117. [CrossRef]

103. Franta, D.; Nečas, D.; Zajíčková, L. Application of Thomas-Reiche-Kuhn sum rule to construction of advanced dispersion models. Thin Solid Films 2013, 534, 432-441. [CrossRef]

104. Franta, D.; Ohlídal, I. Analysis of thin films by optical multi-sample methods. Acta Phys. Slov. 2000, 50, 411-421.

105. Ohlídal, I.; Vohánka, J.; Čermák, M.; Franta, D. Optical characterization of randomly microrough surfaces covered with very thin overlayers using effective medium approximation and Rayleigh-Rice theory. Appl. Surf. Sci. 2017, 419, 942-956. [CrossRef]

106. Franta, D.; Ohlídal, I.; Klapetek, P.; Ohlídal, M. Characterization of thin oxide films on GaAs substrates by optical methods and atomic force microscopy. Surf. Interface Anal. 2004, 36, 1203-1206. [CrossRef] 